Analysis of Rayleigh-Taylor Instability: Statistics on Rising Bubbles and Falling Spikes

C. Kamath, A. Gezahegne, P. Miller

November 2, 2007 
This document was prepared as an account of work sponsored by an agency of the United States government. Neither the United States government nor Lawrence Livermore National Security, LLC, nor any of their employees makes any warranty, expressed or implied, or assumes any legal liability or responsibility for the accuracy, completeness, or usefulness of any information, apparatus, product, or process disclosed, or represents that its use would not infringe privately owned rights. Reference herein to any specific commercial product, process, or service by trade name, trademark, manufacturer, or otherwise does not necessarily constitute or imply its endorsement, recommendation, or favoring by the United States government or Lawrence Livermore National Security, LLC. The views and opinions of authors expressed herein do not necessarily state or reflect those of the United States government or Lawrence Livermore National Security, LLC, and shall not be used for advertising or product endorsement purposes.

This work performed under the auspices of the U.S. Department of Energy by Lawrence Livermore National Laboratory under Contract DE-AC52-07NA27344. 


\title{
Analysis of Rayleigh-Taylor Instability: Statistics on Rising Bubbles and Falling Spikes
}

\author{
Chandrika Kamath, Abel Gezahegne, and Paul L. Miller \\ Lawrence Livermore National Laboratory
}

October 25, 2007

\begin{abstract}
The analysis of coherent structures in Rayleigh-Taylor simulations is a challenging task as the lack of a precise definition of these structures is compounded by the massive size of the datasets. In an earlier work, we used techniques from image analysis to count these coherent structures in two high-resolution simulations, one a large-eddy simulation with 30 terabytes of analysis data, and the other a direct numerical simulation with 80 terabytes of analysis data. Our analysis indicated that there were four distinct regimes in the process of the mixing of the two fluids, starting from the initial linear stage, followed by the nonlinear stage with weak turbulence, the mixing transition stage, and the final stage of strong turbulence. In this paper, we extend our earlier work to focus on only the rising bubbles and the falling spikes. We first consider different ways in which we can constrain the bubble and spike definitions and then extract various statistics on them. Our results on the rising bubble and falling spike counts again show that there are four regimes in the process of fluid mixing, each characterized by an integer-valued slope. Further, the average bubble heights and spike depths are related to similar results obtained using a threshold-based definition. Finally, the ratio of the rising bubbles to all bubbles is very similar in character to the ratio of the falling spikes to all spikes, with near constant values over part of the simulation.
\end{abstract}

\section{Introduction}

Hydrodynamic instabilities such as the Rayleigh-Taylor instability or the Richtmyer-Meshkov instability occur when one fluid is being accelerated by a second fluid. To understand these instabilities, scientists use high-fidelity, three-dimensional computer simulations, which capture the fine-scale detail of the instabilities as they evolve over time. The output of these simulations is often measured in terabytes, requiring semi-automated techniques for their analyses.

In an earlier report [4], we focused on the analysis of the Rayleigh-Taylor instability [6]. This occurs when an initially perturbed interface between a heavier fluid which is on top of a lighter fluid is allowed to grow under the influence of gravity. The fingers of lighter fluid penetrate the heavier fluid in what are referred to as 'bubbles', while 'spikes' of heavier fluid move into the lighter fluid. With time, the bubbles and spikes, which are initially distinct, continue to evolve. In the process, they may grow, split, merge with surrounding bubbles (spikes), or shrink in size relative to other bubbles (spikes) which grow and overtake them.

Our analysis focused on the use of image processing techniques to count the bubble and spike structures and to understand how this count varied over time. Our results showed that there are four distinct regimes in the process of fluid mixing indicated by four regions with 
different slopes. The first stage corresponds to linear growth, where the initial perturbations increase in magnitude but grow independent of each other. In the second non-linear stage, the surface of the bubbles and spikes is no longer single valued and some bubbles/spikes grow faster than the others. The third stage is one of mixing transition, where the fluids are not quite mixed. The final stage is one of strong turbulence, leading to well-mixed fluid.

In this paper, we extend our earlier work to focus on the rising bubbles, and correspondingly, the "falling" spikes. We first briefly summarize the data and our earlier analysis approach in Section 2. Next, in Section 3, we describe the different ways in which we can define a rising bubble. In Section 4, we present several statistics on the bubbles and spikes using these different techniques. These statistics include the counts for the coherent structures, the slopes of the different regimes in the count curves, the average height of the structures, the ratio of the rising bubbles to all bubbles, and correspondingly, the falling spikes to all spikes. We conclude in Section 5 with a summary of our work.

In the rest of this paper, we will use the term "rising bubbles" to include the "falling spikes", as well. The two types of structures will be referred to separately only when necessary.

\section{Description of the data}

We consider two high-fidelity simulations in our analysis - one a large-eddy simulation (LES), and the other a direct numerical simulation (DNS) $[2,1]$, both generated using the Miranda code at Lawrence Livermore National Laboratory. The datasets simulate the problem where the heavier fluid of density 3 is on top of the lighter fluid of density 1 . The three-dimensional computational domain is a cube with uniformly-spaced grid points, periodic boundary conditions in $x$ and $y$, and no-slip walls imposed in $z$ at the top and bottom of the cube. Both the LES and the DNS datasets are partitioned in vertical columns corresponding to the number of processors used to run the simulation. Each column is written out to a single file.

The data from the LES simulation is on an $1152^{3}$ grid, with the initial interface perturbed as described in [2]. This simulation saved flow fields from 759 time steps. At each time step, seven variables are output in single precision at each grid point. These include the pressure, the density, the $x, y$, and $z$ velocities, the viscosity, and the diffusivity. The total size of the analysis data is 30 terabytes.

The second data set from the DNS simulation under is run under identical physical conditions to the LES simulation [1]. The grid is larger, at $3072^{3}$, and the simulation saved flow fields from 248 time steps. Only five variables are output in single precision at each time step: the pressure, the density, and the $x, y$, and $z$ velocities. The total size of the analysis data is 80 terabytes.

We note that the sample times at which the flow fields are saved for the LES and the DNS simulations are not physically or numerically equivalent.

\subsection{Summary of earlier analysis}

In our earlier analysis [4], we focused on identifying the bubble and spike structures in the LES and DNS output. To make the analysis of these massive datasets tractable, we first reduced the three-dimensional data to two dimensions and then identified the coherent structures in these two-dimensional images. These images are also used in the current analysis for counting the rising bubbles. We next describe them briefly; details are available in our earlier work [4]. 


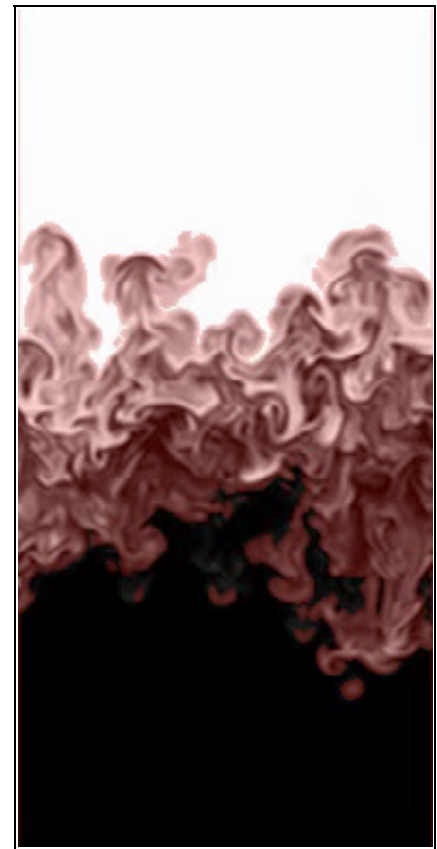

(a)

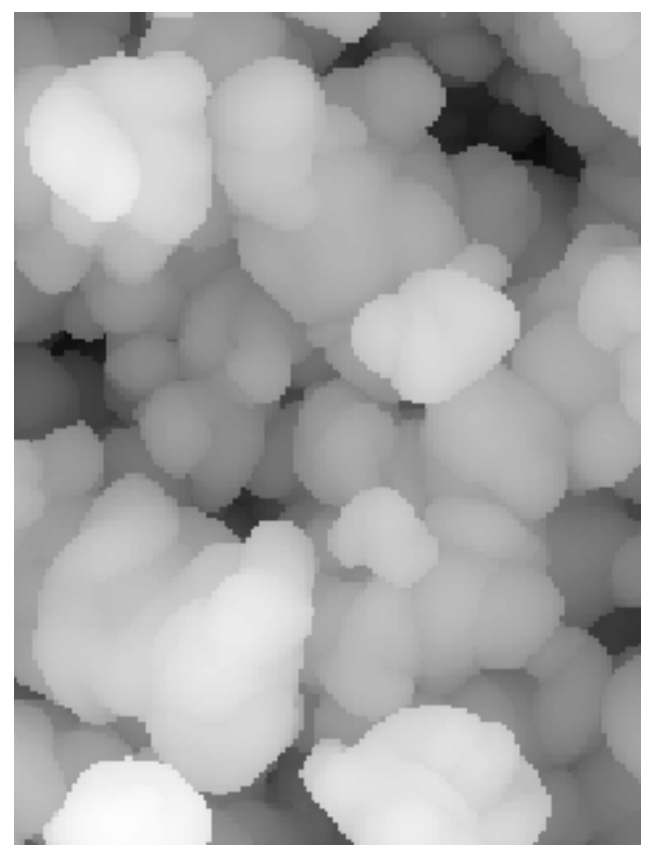

(b)

Figure 1: Defining a bubble in 3-D. (a) The results of the 3-D region growing segmentation (in pink) superposed on the original image. The image is the $x=1$ slice from the central $144 \times 192$ grid of time step 350 of the LES data. The image is cropped in the $z$ direction to show only the region around the interface. (b) The top view of the bubbles for the $144 \times 192$ grid, where the intensity of a pixel reflects its height from the initial fluid interface. 
To reduce the three-dimensional data, we first used a simple region growing method to identify the boundary of the bubble. Starting with grid points at the top of the cube, we grow a region downward by adding a grid point to the region if it is 6-connected to the region and its intensity, along with the intensities of all of its 6 neighbors, is greater than a high threshold. This threshold is related to the density of the heavier fluid. The 6 neighbors considered are 1 grid point away in the $x, y$, and $z$ direction from the grid point under consideration. A similar region grown from the bottom of the cube, with a threshold based on the density of the lighter fluid, determines the boundary of the spikes. Figure 1 shows the results of the 3 -D region growing technique on the density variable of the LES dataset at time step 350. The image shown is a two-dimensional slice of the three-dimensional data. The pixels in pink highlight the mixing region and the boundary pixels form the boundary of the bubble and spike structures. Note that in three-dimensions, this boundary can be quite complex.

Next, to convert this three-dimensional boundary into a two-dimensional image, we consider the top and bottom views of the mixing region which is between the bubble and spike boundaries. The image formed by taking the height of each pixel at the bubble boundary, from the initial interface between the two fluids, is shown in Figure 1, panel (b). The bubble height (spike depth) is the height (depth) of the first boundary pixel encountered along the positive (negative) $\mathrm{z}$ axis as we move towards the fluid interface starting at the top (bottom) of the cube. Note that there may be more than one boundary pixel along $z$ for each $(x, y)$ location. For the bubbles, we select the one which is closest to the top of the cube and similarly, for the spikes, we select the one which is closest to the bottom of the cube. We refer to these images as the height-depth maps (HDM) as they reflect the height (depth) of the bubbles (spikes) from the original interface.

In addition to the height-depth maps, we also found it very useful to consider the images formed by the other simulation variables at the bubble/spike boundaries. Figures 2 and 3 show these images for the bubbles for the LES and DNS data, respectively. These variables include the pressure, the velocities in the $x, y$, and $z$ directions, the height-depth map image and the magnitude of the $x-y$ velocity. The images are of the central $144 \times 192$ and $300 \times 300$ grid points of the LES and DNS data, respectively.

In our earlier work we showed how we can exploit the HDM and the magnitude of the $x-y$ velocity to identify the tips of all bubbles. The magnitude of the $x-y$ velocity is small at the center of the bubble (the bubble tip region) and around the perimeter of the bubbles as seen in the top view. However, the pixels in the perimeter are in a region where the height varies considerably, while the pixels in the center have roughly constant height. This observation allows us to identify regions at the bubble tips which have a low magnitude of the $x-y$ velocity and roughly constant height. The centroid of such a region (or the highest point in the region) can then be considered to be the tip of a bubble.

\section{Defining a rising bubble}

Instead of counting all the bubbles, as we did in our earlier work, we could constrain the count to just the rising bubbles as one would expect them to be responsible for the growth of the mixing layer. In related work, Kartoon et al. [5] define a rising bubble in the Rayleigh-Taylor instability as one which is accelerating, while a decelerating bubble is considered to be sinking. However, for their analysis of the Richtmyer-Meshkov instability, they use a velocity-based criterion as in the work of Gardner et al. [3]. This defines a rising (sinking) bubble as one with a positive (negative) velocity. 

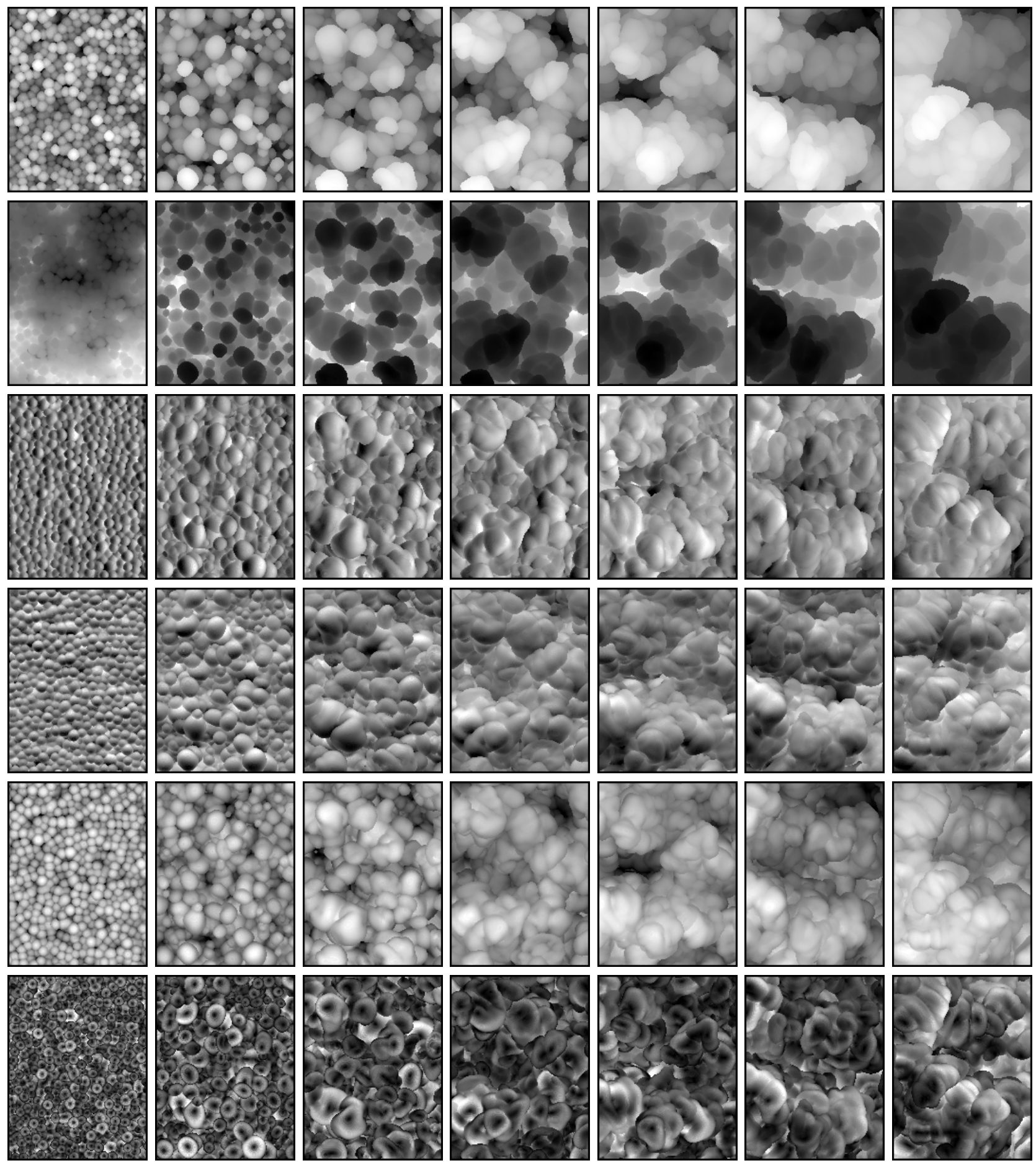

Figure 2: The other variables at the bubble boundary for the central $144 \times 192$ grid every 100-th time step, starting at time step 100, for the LES data. Each image is normalized so the intensity values lie between 0 and 255. The columns correspond to the time steps. The rows are, from top to bottom, the HDM, the pressure, $x$ velocity, $y$ velocity, $z$ velocity, and the magnitude of the $x-y$ velocity. 

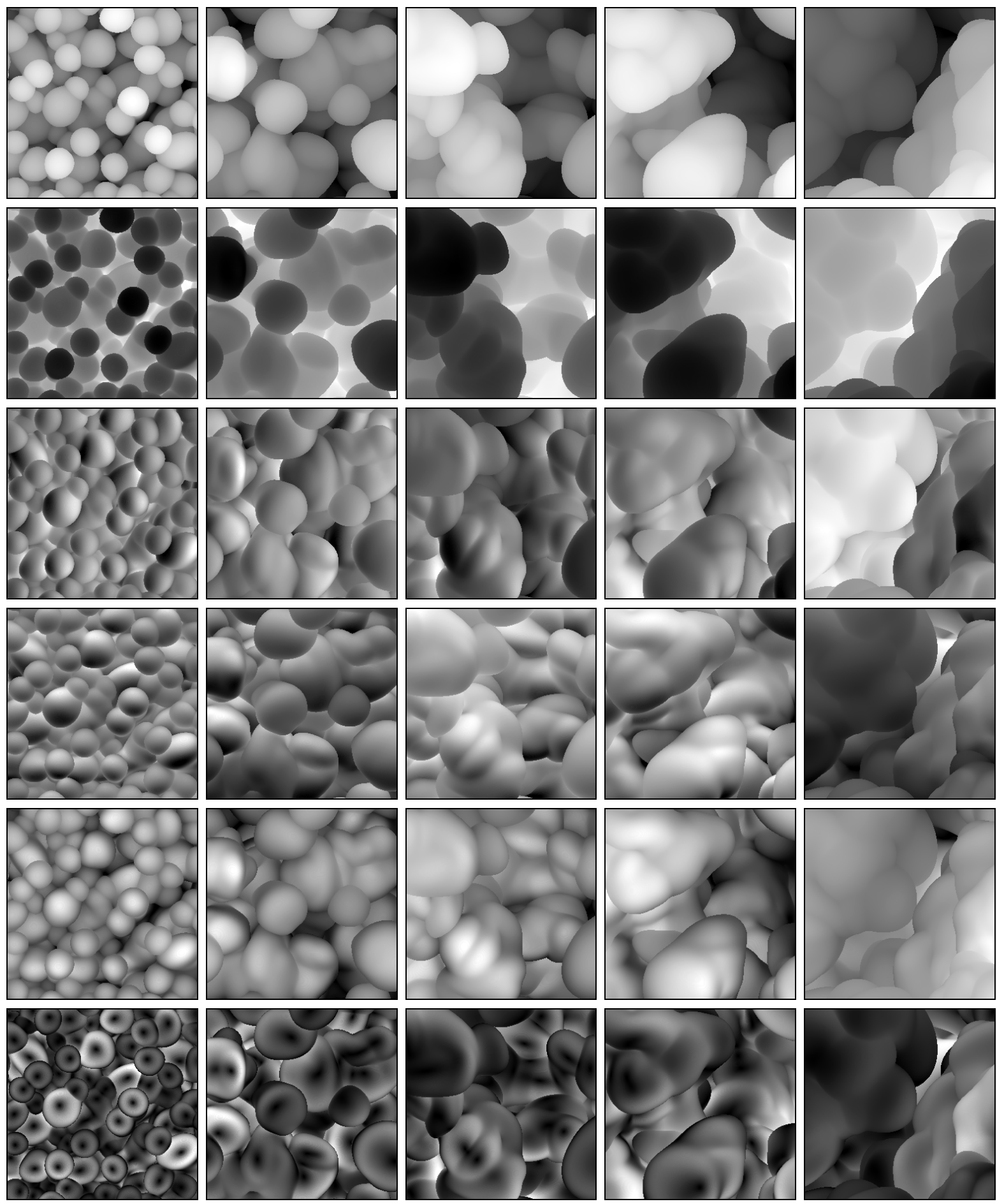

Figure 3: The other variables at the bubble boundary for the central $300 \times 300$ grid every 50-th time step, starting at time step 50 and ending at 247, for the DNS data. Each image is normalized so the intensity values lie between 0 and 255. The columns correspond to the time steps. The rows are, from top to bottom, the HDM, the pressure, $x$ velocity, $y$ velocity, $z$ velocity, and the magnitude of the $x-y$ velocity. 


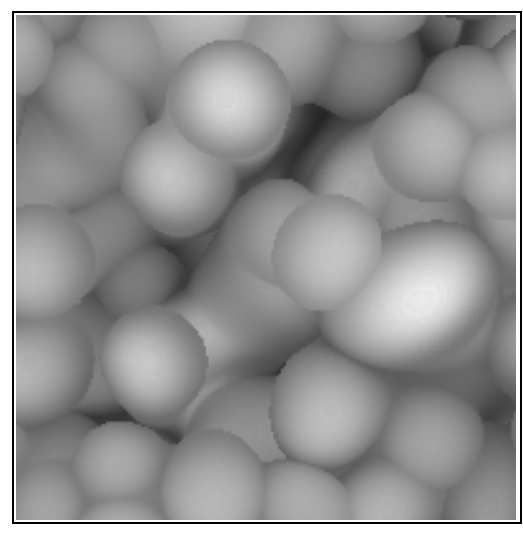

(a)

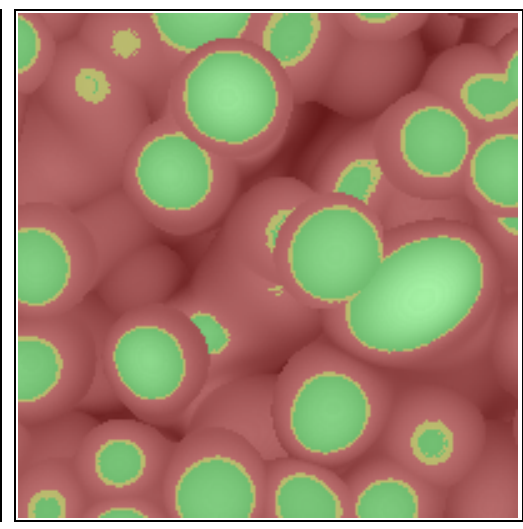

(b)

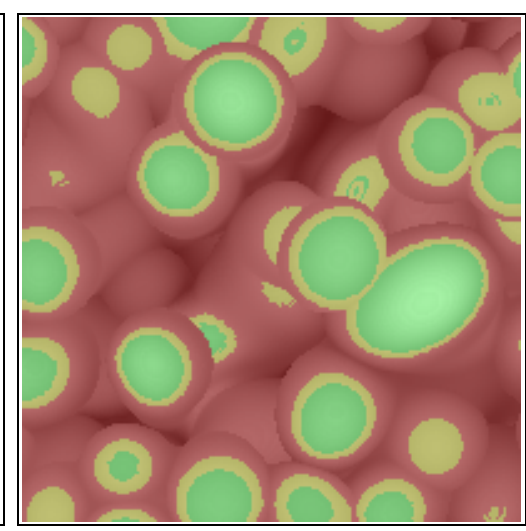

(c)

Figure 4: Identifying regions with positive $z$-velocity. (a) A $200 \times 200$ subset of the $z$-velocity image from the DNS simulation at time step 50. (b) The subset with values highlighted as follows - red: $z$-velocity below -0.1 ; green: $z$-velocity above 0.1 ; yellow: $z$-velocity between -0.1 and 0.1. (c) Similar to panel (b), but with the threshold set to 0.3. Note the bubble in the lower right corner which now does not have any green pixels.

In this paper, we consider several velocity-based definitions of rising bubbles. We need to address two issues - how do we define the "velocity of a bubble" and what threshold do we use to restrict this velocity so we identify only the rising bubbles?

Based the description of the data in Section 2, we have access to the $x-, y$-, and $z$-velocities at the bubble boundaries in two dimensions. In counting the rising bubbles, it seems appropriate to focus on the $z$-velocity to separate the rising bubbles from the bubbles which are not rising. However, there is a large variation in the values of the $z$-velocity over the bubble. For example, in Figure 4, panel (a), we show the $z$-velocity of a $200 \times 200$ subset of the bubbles at time step 50 of the DNS data. Brighter values indicate a higher $z$-velocity. At this time-step, the bubbles are clearly defined. For the bright, oval-shaped bubble in the middle right of the image, the $z$-velocity near the center is close to 3.0, and rapidly reduces to around -1.5 near the perimeter of the oval. These negative values around the perimeter of a bubble are the rule rather than the exception, as seen in Figure 4, panel (b), where the pixels with values lower than -0.1 are highlighted in red. Given this wide variation in the $z$-velocity over the bubble, we first need to address the issue of identifying the pixels whose $z$-velocity values can be used to define the velocity of a bubble. We investigated several options:

- Consider the velocity of the bubble to be the average of the $z$-velocity values of all pixels which form a bubble. Unfortunately, as explained in our earlier report [4], the extent of a bubble can be clearly identified only at the early time steps. So, this approach is not practical for calculating bubble velocities at later time. In addition, this definition includes both the positive velocities near the center of the bubble and negative velocities near the perimeter. It is unclear if averaging these quantities is the appropriate way to calculate the velocity of the bubble as a whole.

- Consider the velocity of the bubble to be the $z$-velocity at the bubble tip. Recall that we first identify the bubble tip regions using the magnitude of the $x-y$ velocity and the HDM. We then have several ways of selecting the pixel which represents the tip of the bubble. It can either be the centroid of the region (which may not have a physical meaning) or it 
can be the pixel with the highest height or the largest $z$-velocity in the region. Regardless of how we select the pixel to represent the bubble tip, there are several issues with using the $z$-velocity at this pixel to represent the $z$-velocity of the bubble. First, the definition of the bubble velocity is not very robust as it is defined at a single pixel. The location of this tip pixel can vary somewhat across consecutive time steps, especially when several distinct regions close to each other have low values for the magnitude of the $x-y$ velocity. This makes the $z$-velocity at the tip pixel rather noisy, resulting in a bubble not being consistently counted as a rising bubble over time. Second, the definition is sensitive to how the tip pixel is defined when two bubbles merge or split.

We also considered several choices for the $z$-velocity threshold to identify the rising bubbles. One option is to use a hard, data-independent threshold for all time steps and consider all bubbles with $z$-velocity greater than say, zero, as rising bubbles. Alternately, we could use a threshold which varies with time and is defined in terms of the data. For example, we could consider all bubbles with a $z$-velocity greater than the mean $z$-velocity to be rising bubbles. This again brings up the issue of which pixels to use in calculating the mean $z$-velocity. Using all the pixels in the $z$-velocity image is not appropriate as it represents just the top view of the bubble boundary. If we use only the bubble tips, the mean is noisy and not very well behaved, especially at the later time steps when there are few bubbles.

Our experimentation with these options indicated that a stable definition of a rising bubble seemed unlikely if we first identified all the bubbles and then determined which ones were rising. This led us to investigate a different approach. We start by assuming that if all the pixels which comprise a bubble in the two dimensional image have negative $z$-velocity, then, it cannot be a rising bubble. Thus, for a bubble to be considered as a rising bubble, it must have at least one pixel with a positive $z$-velocity. To ensure that the results are not adversely affected by pixels with $z$-velocity slightly above or slightly below zero, we remove pixels with velocities in the range $\left[-T_{z}, T_{z}\right]$ from further consideration, where the threshold $T_{z}$ is a small positive value.

For example, Figure 4, panels (b) and (c), show the pixels (in green) which have a positive $z$-velocity using a threshold of 0.1 and 0.3 , respectively. Note that at the threshold of 0.1 , there are relatively few pixels in the range $[-0.1,0.1]$ as indicated by the yellow pixels. When the threshold is increased to 0.3, this number increases as expected, and some bubbles which were connected to a neighboring bubble before (such as the oval one in the middle right of the image), are now no longer connected. Also some bubbles, such as the one in the lower right corner, no longer have any green pixels. To avoid very small green regions from influencing further processing, we ignore regions smaller than 10 (3) pixels in size for the DNS (LES) data sets.

Figure 5 shows the positive and negative $z$-velocity pixels displayed on the log of the magnitude of the $x-y$ velocity image for a $600 \times 600$ sub-image at time steps $50,100,150$, and 200 for the DNS data. We observe that in these images, the green regions of positive velocity occur near the tip of a bubble; they do not cover the bubble entirely, staying well within the perimeter of the bubble. Therefore, if the green region has pixels with a low magnitude of the $x-y$ velocity, then those pixels are the ones at the tip, not the outer perimeter of the bubble (which usually has a negative $z$-velocity and therefore, does not lie in the green region). This allows us to define a rising bubble region as one which has a positive velocity and contains at least one pixel with a low magnitude of the $x-y$ velocity. Each such region is then counted as a rising bubble.

We note that this definition does not necessarily define the extent of each bubble. The green regions shown in Figure 5 cover almost all the area of a rising bubble at early time. However, 
as the bubbles grow and have more complicated boundaries (as seen in the top view), we may have green regions which appear unconnected, but are part of the same bubble. Not all of these regions will have the pixels with a low magnitude of the $x-y$ velocity. Only the region which satisfies this constraint is counted as a rising bubble.

Figure 6 shows a subset of the DNS data at time steps 50, 55, 60, and 65. Green pixels which also have a low magnitude of $x-y$ velocity (less than 0.1 ) are highlighted in dark blue. Any green region with one or more such pixels is considered to be a rising bubble. These images show several aspects of the bubble behavior. Some bubbles, such as the three in the middle right of the sub-image, don't change much in size, but have shrinking green regions, which first become completely yellow, and then shrink further to become completely red. Others, such as the one near the top, grow and push aside neighboring bubbles, which are swept downstream. Though these images are taken five time steps apart, it is clear that the change in the green region occupied by a rising bubble over consecutive time steps is small enough that we can use it to effectively track the rising bubbles over time. We observe that this would allow us to understand the bubble dynamics and to obtain statistics on the behavior of the bubbles, both individually, as well as collectively over all the bubbles. These statistics could include the length of bubble lifetimes as well as the changes in the height and velocity over time. Further insights could also be obtained on how bubbles split and merge as they evolve over time.

\section{Statistics on rising bubbles and falling spikes}

We next present various statistics for the rising bubbles and falling spikes for the LES and DNS data sets. These statistics include the counts for the coherent structures, the slopes of the different regimes in the count curves, the average height of the structures, the ratio of the rising bubbles to all bubbles, and correspondingly, the falling spikes to all spikes.

Recall that we first identify rising pixels by thresholding on the $z$-velocity. This identifies regions with a positive $z$-velocity near the tip of a bubble and excludes pixels near the bubble perimeter which have a negative $z$-velocity. Next, any such region which contain at least one pixel with a small value of the magnitude of the $x-y$ velocity is counted as a rising bubble. We use the 8-neighbor connected component labeling algorithm from image processing [7] to identify the pixels which form a region.

The color images shown in this section are to be interpreted as follows. If the $z$-velocity threshold is chosen as $T_{z}$, then pixels in green have $z$-velocity higher than $T_{z}$, pixels in red have $z$-velocity lower than $-T_{z}$, and pixels in yellow have $z$-velocity in the range $\left[-T_{z}, T_{z}\right]$. If the threshold on the magnitude of the $x-y$ velocity is chosen as $T_{x-y}$, then pixels in blue are those whose $z$-velocity is greater than $T_{z}$ and whose magnitude of the $x$ - $y$ velocity is less than $T_{x-y}$. We next discuss how we choose the two thresholds, $T_{z}$ and $T_{x-y}$.

\subsection{Choice of threshold $T_{z}$}

The threshold on the $z$-velocity, $T_{z}$, is selected to be a small positive value. The intent is to identify the region around the bubble tip while still remaining within the perimeter. We also need to ensure that our choice of the threshold does not lead to two distinct bubbles appearing to be connected because they have positive $z$-velocity pixels which are eight-neighbors of each other. For example, in Figure 7, panel (a), we show several bubbles whose positive $z$-velocity areas are connected. In this example, which shows the DNS bubble data at time step 50, we use $T_{z}=0.1$ and $T_{x-y}=0.15$. When we increase $T_{z}$ to 0.2 , as shown in panel (b), the yellow 


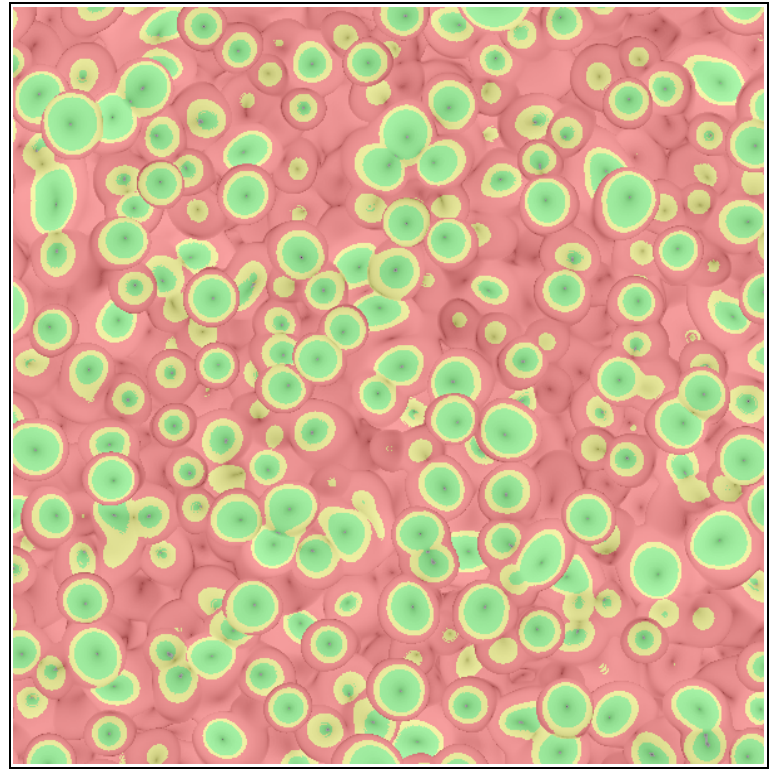

(a)

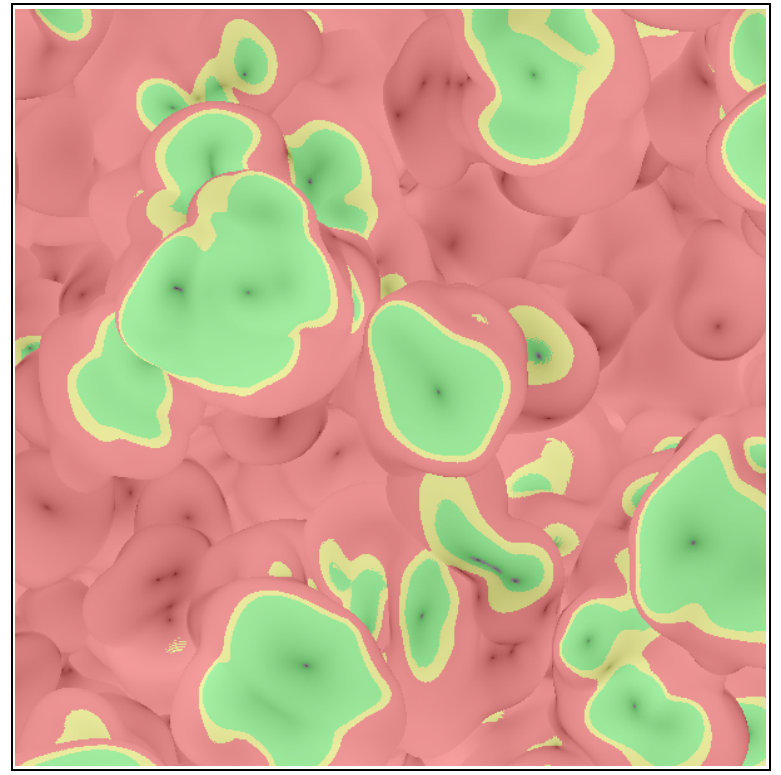

(c)

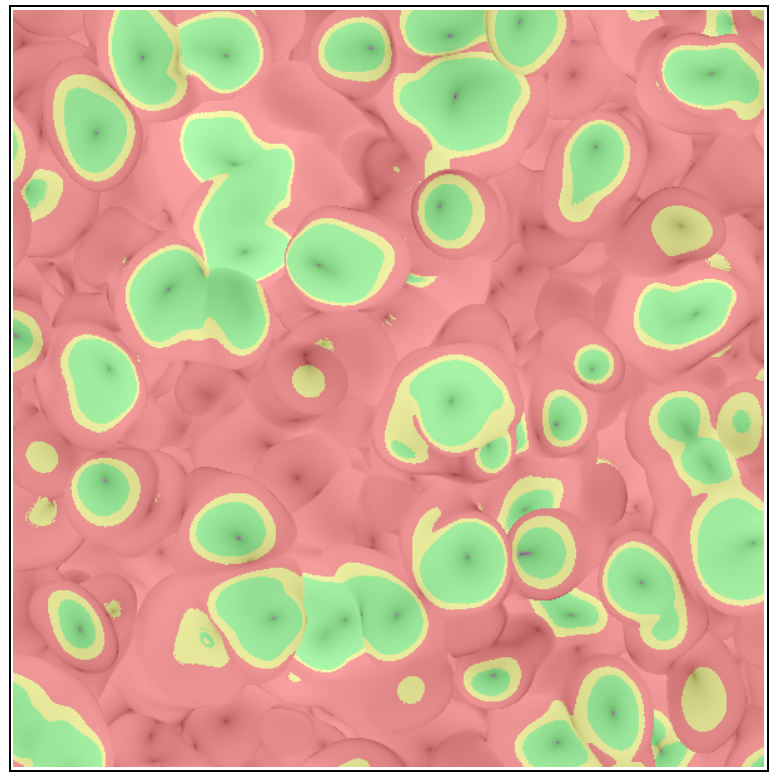

(b)

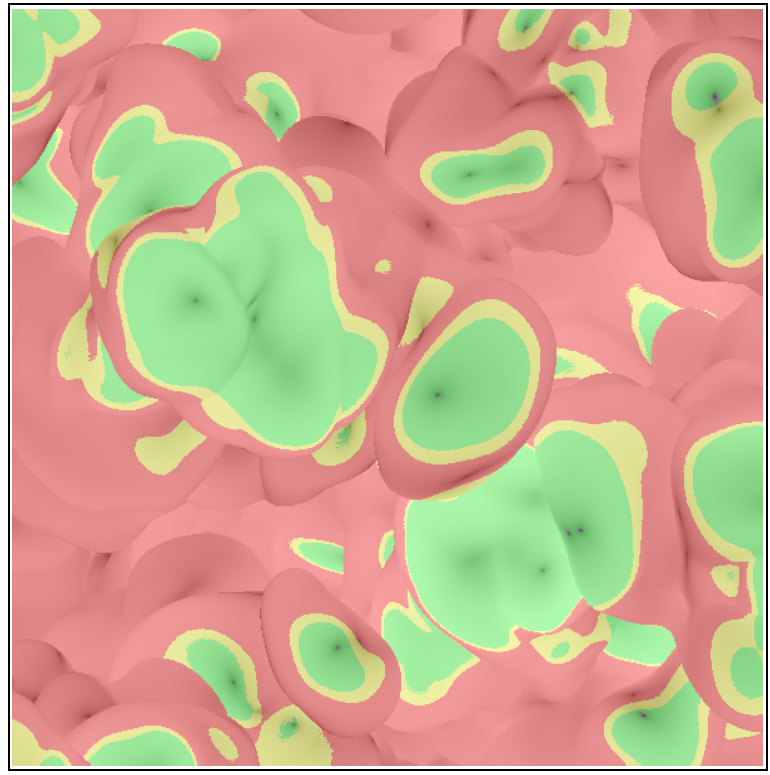

(d)

Figure 5: Areas of positive and negative $z$-velocity displayed on the log of the magnitude of $x-y$ velocity image for a $600 \times 600$ subset of the DNS data at time steps (a) 50, (b) 100 , (c) 150, and (d) 200. Pixels in red have $z$-velocity less than -0.3, those in green have $z$-velocity greater than 0.3 , and the pixels in yellow have values in the range $[-0.3,0.3]$. 


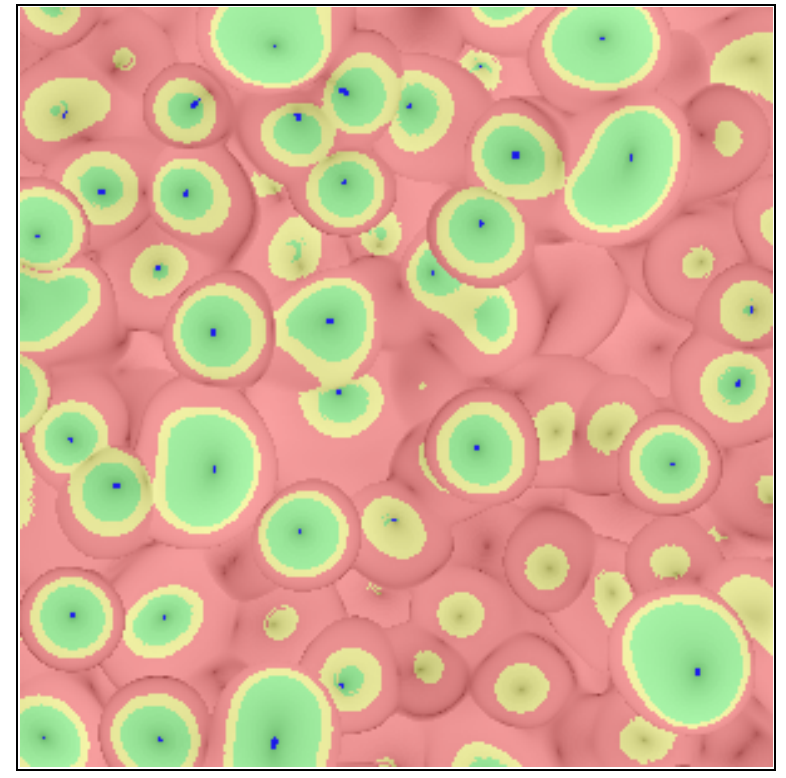

(a)

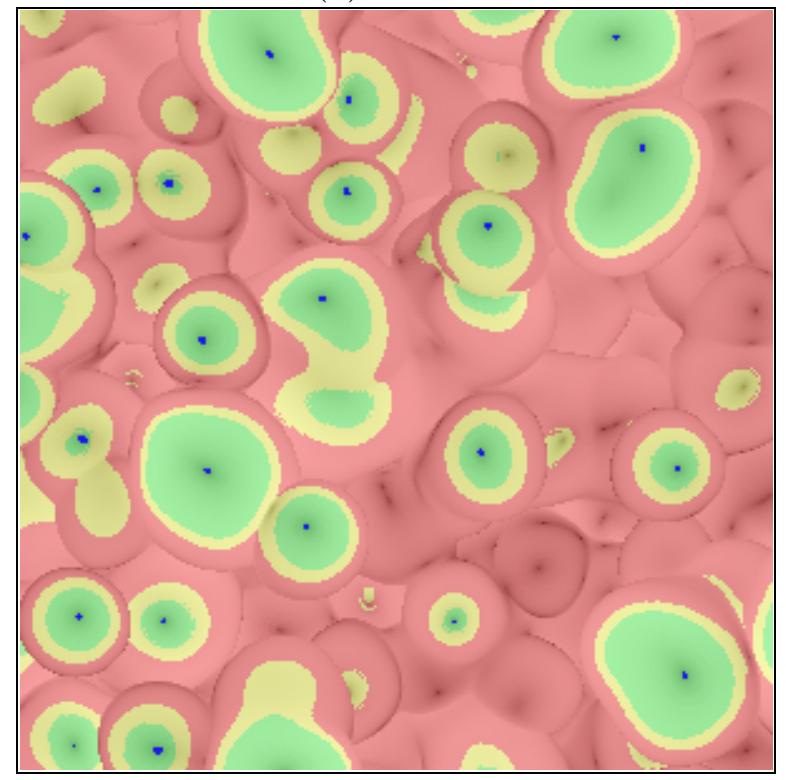

(c)

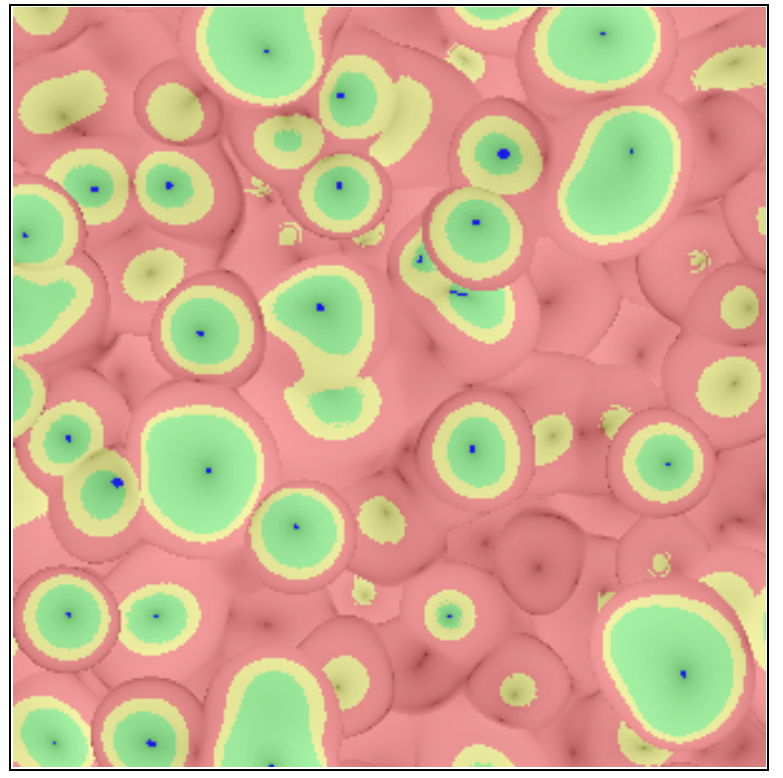

(b)

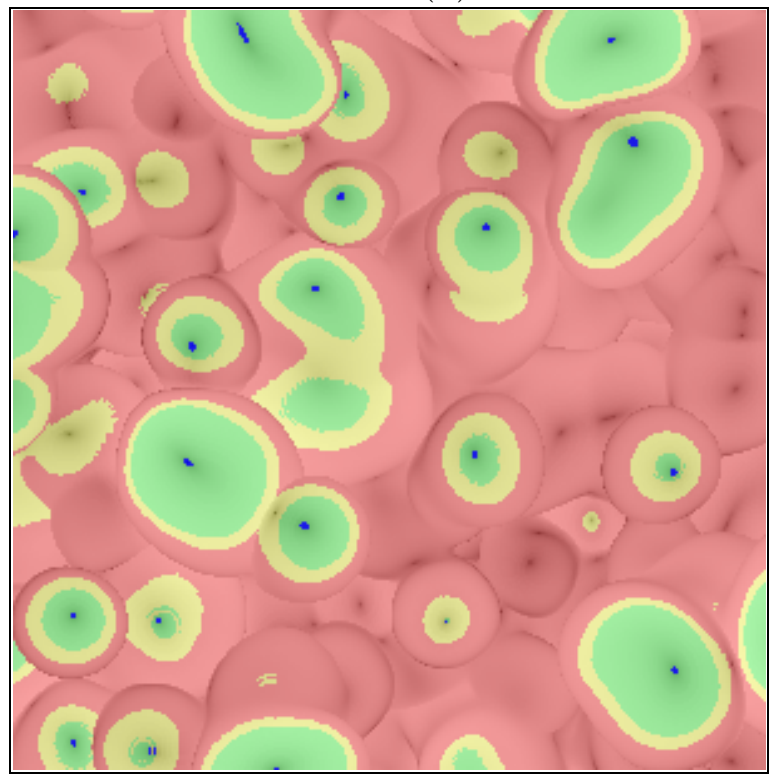

(d)

Figure 6: Areas of positive and negative $z$-velocity displayed on the log of the magnitude of $x-y$ velocity image for a $300 \times 300$ subset of the DNS data at time steps (a) 50, (b) 55, (c) 60, and (d) 65. Pixels in red have $z$-velocity less than -0.3 , those in green have $z$-velocity greater than 0.3 , and the pixels in yellow have values in the range $[-0.3,0.3]$. Green pixels with a low value of the magnitude of the $x-y$ velocity (less than 0.1 ) are highlighted in blue. 


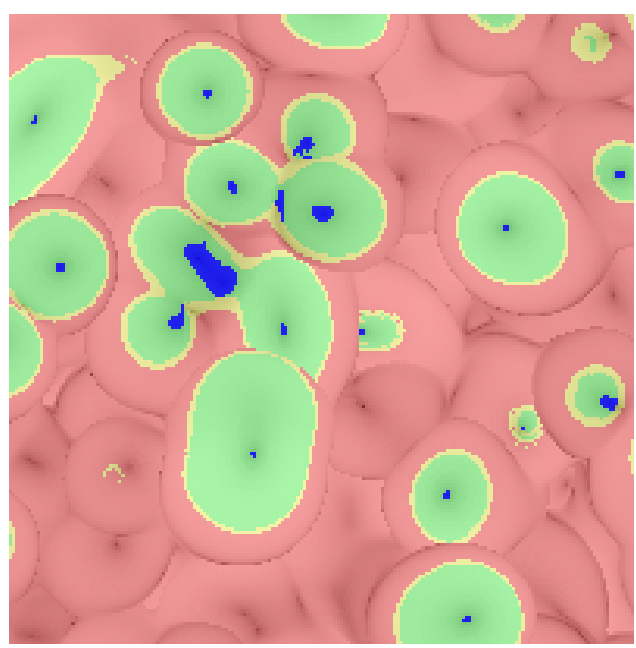

(a)

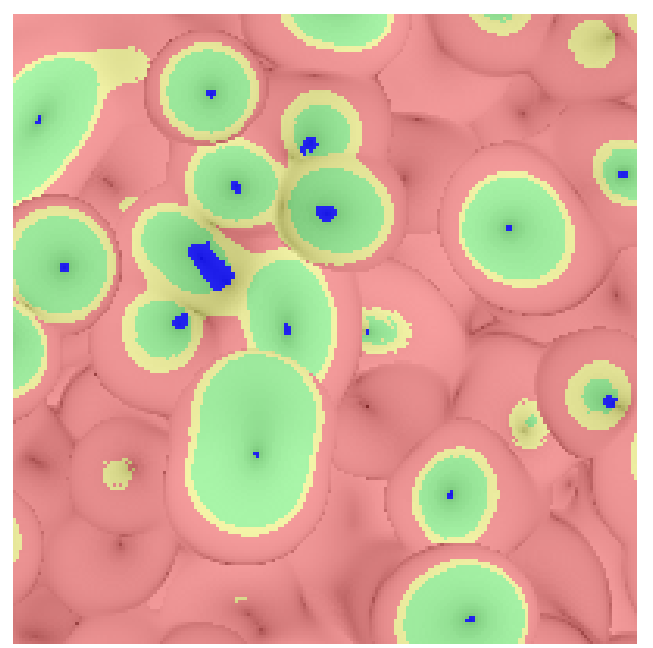

(b)

Figure 7: Effect of the choice of $T_{z}$ shown using the DNS bubble data at time step 50 with $T_{x-y}=0.15$. (a) $T_{z}=0.1$ and (b) $T_{z}=0.2$.

regions increase in size and the bubbles are no longer connected. However, this could also result in some bubbles being no longer counted as their green region has shrunk and does not overlap pixels with a low magnitude of the $x-y$ velocity. This is the case for the small bubble near the middle right of the image. The overall effect of this is to change the time step at which a bubble stops being counted as a rising bubble.

The lower resolution of the LES data, and correspondingly, the smaller size of the structures, highlights another issue with the choice of $T_{z}$. The images in Figure 8, panels (a) and (b), show that when we increase the values of $T_{z}$ from 0.1 to 0.2 , the yellow regions may grow to completely cover a bubble, resulting in several bubbles not being counted at all. We do not have this problem in the DNS data as the structures are larger and therefore less sensitive to the choice of the threshold. Further complicating the issue is the fact that the bubble and spike $z$-velocities are different. For example, Figure 8, panel (c), shows the results for the spikes using the same thresholds as for the bubbles in panel (b). We notice that unlike the bubbles, we are able to count most of the spikes.

These observations indicate that we need to choose the threshold $T_{z}$ with care as there are several competing considerations. The original idea behind choosing $T_{z}$ to be a small positive value was to exclude pixels with $z$-velocity very near zero. When we consider $T_{z}=0.1$, we find that it leads to a narrow yellow region around the bubbles and spikes, usually a pixel or two in width. This may cause some bubbles to appear connected. Therefore, a value higher than $T_{z}=0.1$ seems appropriate. However, if we increase it too much, especially in the case of the lower resolution LES data, it could result in completely missing bubbles as shown in Figure 8, panel (b). Further, since the $z$-velocity is different for the bubbles and spikes, we may need to select different thresholds for the two coherent structures.

We next experimented with various values for $T_{z}$ for the bubbles and spikes for the LES and DNS datasets. For these studies, we set the threshold $T_{x-y}=0.15$ as it was large enough to identify most of the bubbles and spikes at early time. Figures 9 and 10 show the resulting rising bubble and falling spike count for the DNS and LES datasets, respectively. These plots indicate that the DNS data is not sensitive to the choice of the $z$-velocity threshold. This is to 


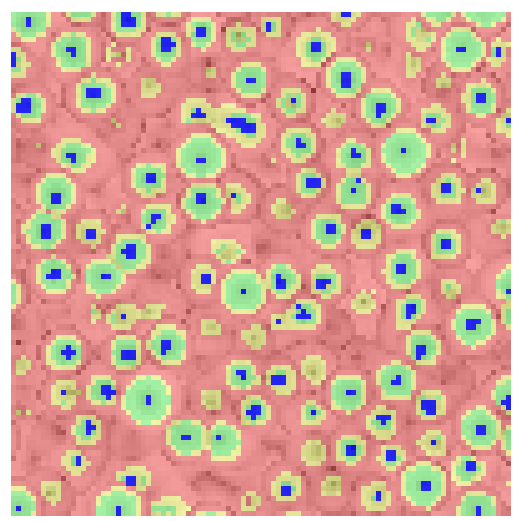

(a)

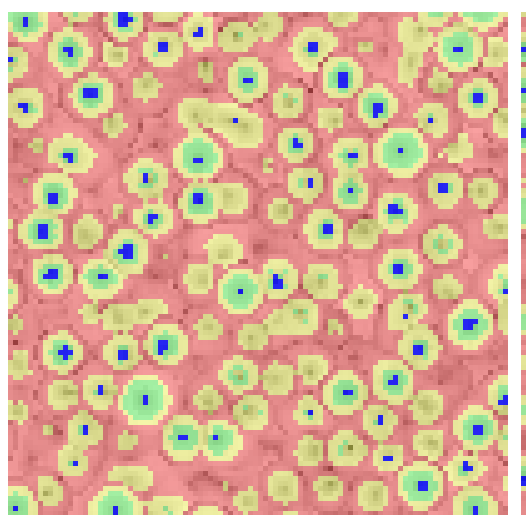

(b)

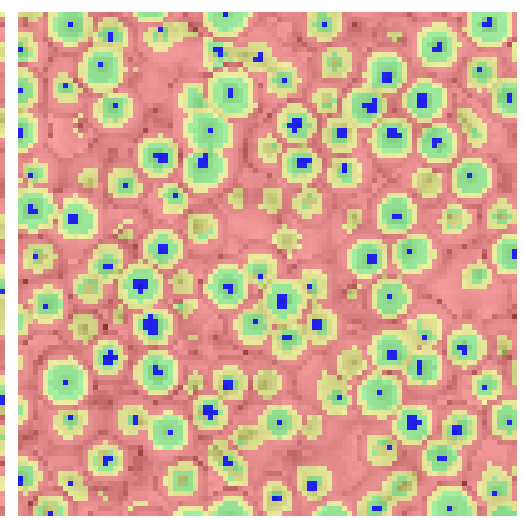

(c)

Figure 8: Effect of the choice of $T_{z}$ shown using the LES data at time step 100 with $T_{x-y}=0.15$. (a) Bubble data with $T_{z}=0.1$, (b) Bubble data with $T_{z}=0.2$, and (c) Spike data with $T_{z}=$ 0.2 .

be expected given its higher resolution and, correspondingly, the larger size of its structures. On the other hand, the results for the LES data are sensitive to the choice of the $z$-velocity threshold, especially at early time when the structures of interest are small. As we have observed in Figure 8, panel (b), at $T_{z}=0.2$, several bubbles in the LES data are not counted as they are completely covered by the yellow region. For this threshold, the problem is not as severe for the spikes (Figure 8, panel (c)), a fact reflected in the plots as well. At later time, when the structures are larger, the counts are far less sensitive to the choice of $T_{z}$.

Based on these plots, we select the $z$-velocity threshold, $T_{z}$, to be 0.15 for both bubbles and spikes for the LES and DNS data sets.

\subsection{Choice of threshold $T_{x-y}$}

The threshold on the magnitude of the $x-y$ velocity is chosen by considering its values near the center of the bubbles and spikes especially at the early time steps. We have observed that there is usually a single pixel or two at the bubble tip with a value lower than the surrounding pixels. By selecting a threshold which is just above this minimum, we can correctly identify most of the bubbles at early time. If however, the threshold is just below this minimum, we miss counting many clearly identifiable bubbles.

Figures 11 and 12 show the effects of varying the $T_{x-y}$ threshold on the rising bubble and falling spike counts for the DNS and LES data sets, respectively. They show that when the threshold $T_{x-y}$ is varied from 0.125 to 0.2 , keeping the threshold $T_{z}$ constant at 0.15 , the number of structures counted does not change much. At values below 0.125, such as $T_{x-y}=0.1$, we miss many of the structures for DNS spikes and LES bubbles and spikes. As we increase the threshold, some structures which had been missed earlier as they were just below the threshold, are now counted. Also, the number of pixels which meet the criteria in each structure increases, but as a region is counted if it has at least one such pixel, having additional pixels does not affect the count.

Based on these plots, we select the $z$-velocity threshold, $T_{x-y}$, to be 0.15 for both bubbles and spikes for the LES and DNS data sets. For the rest of the paper, will assume that the statistics on the rising bubbles and falling spikes are obtained for the case when the two thresholds, $T_{z}$ and $T_{x-y}$, are both set to 0.15 . 


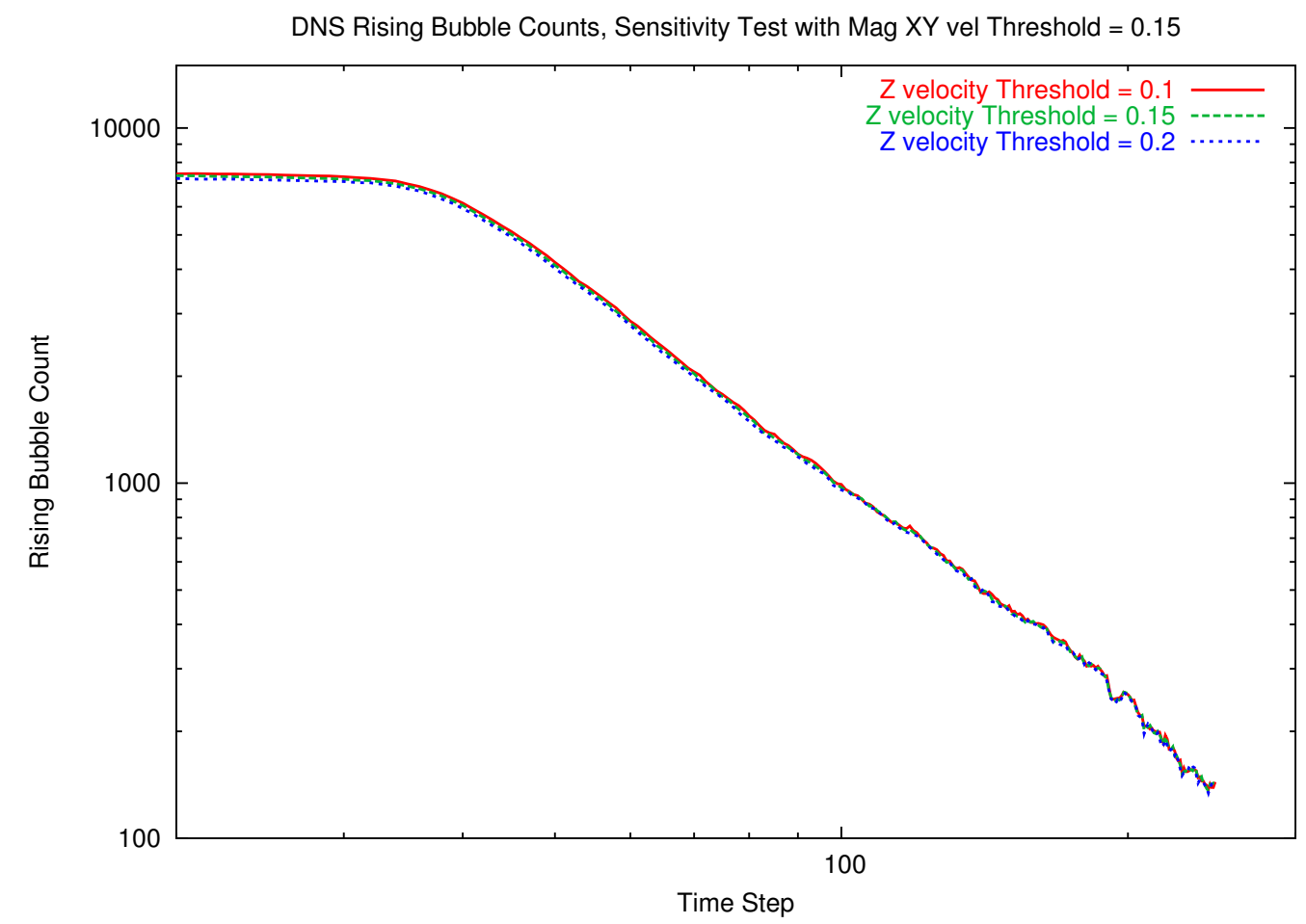

(a)

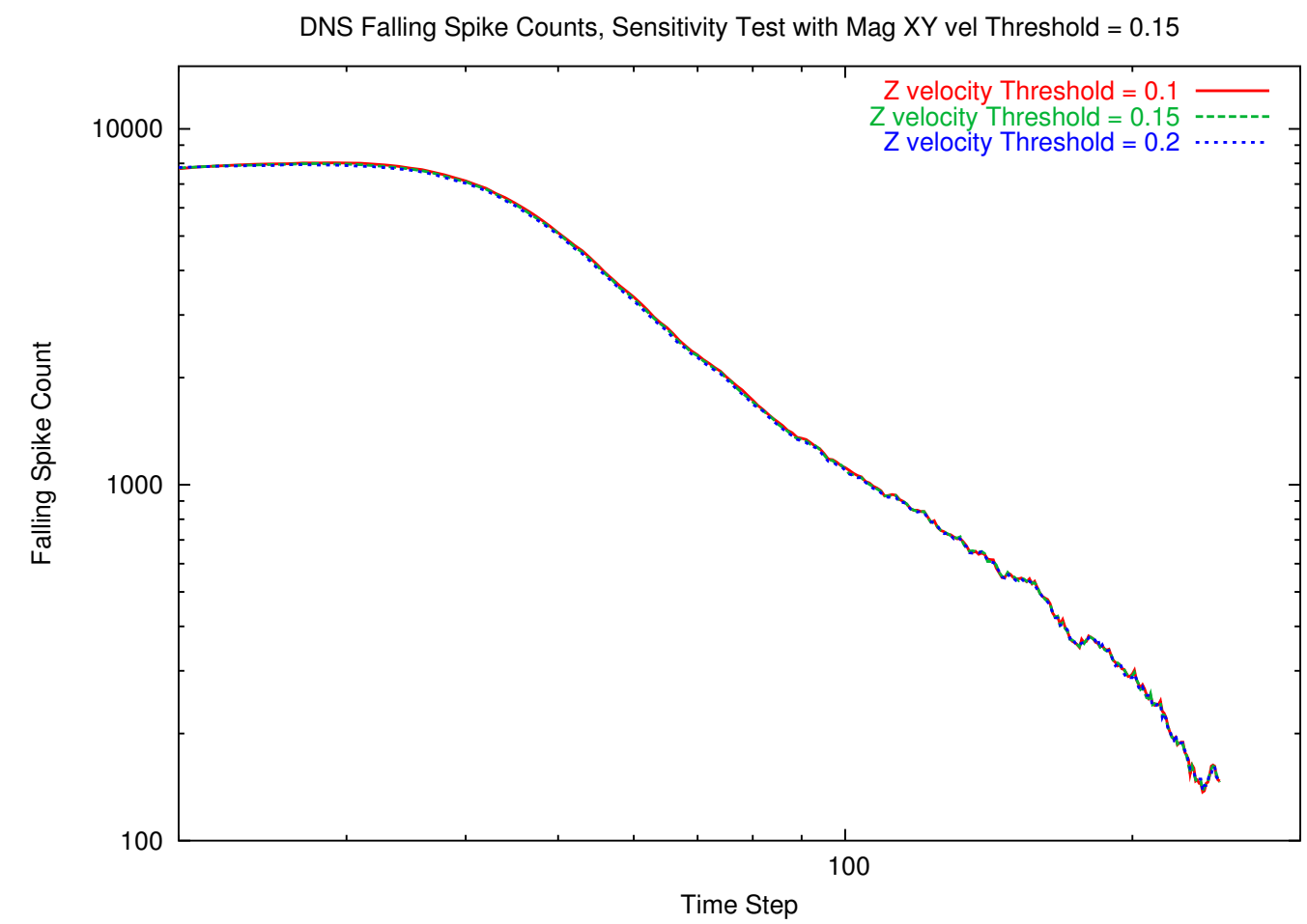

(b)

Figure 9: Effect of varying the $z$-velocity threshold on the rising bubble and falling spike counts for the DNS data. The threshold on the magnitude of the $x-y$ velocity is set to 0.15 . (a) Rising bubble count and (b) falling spike count. 


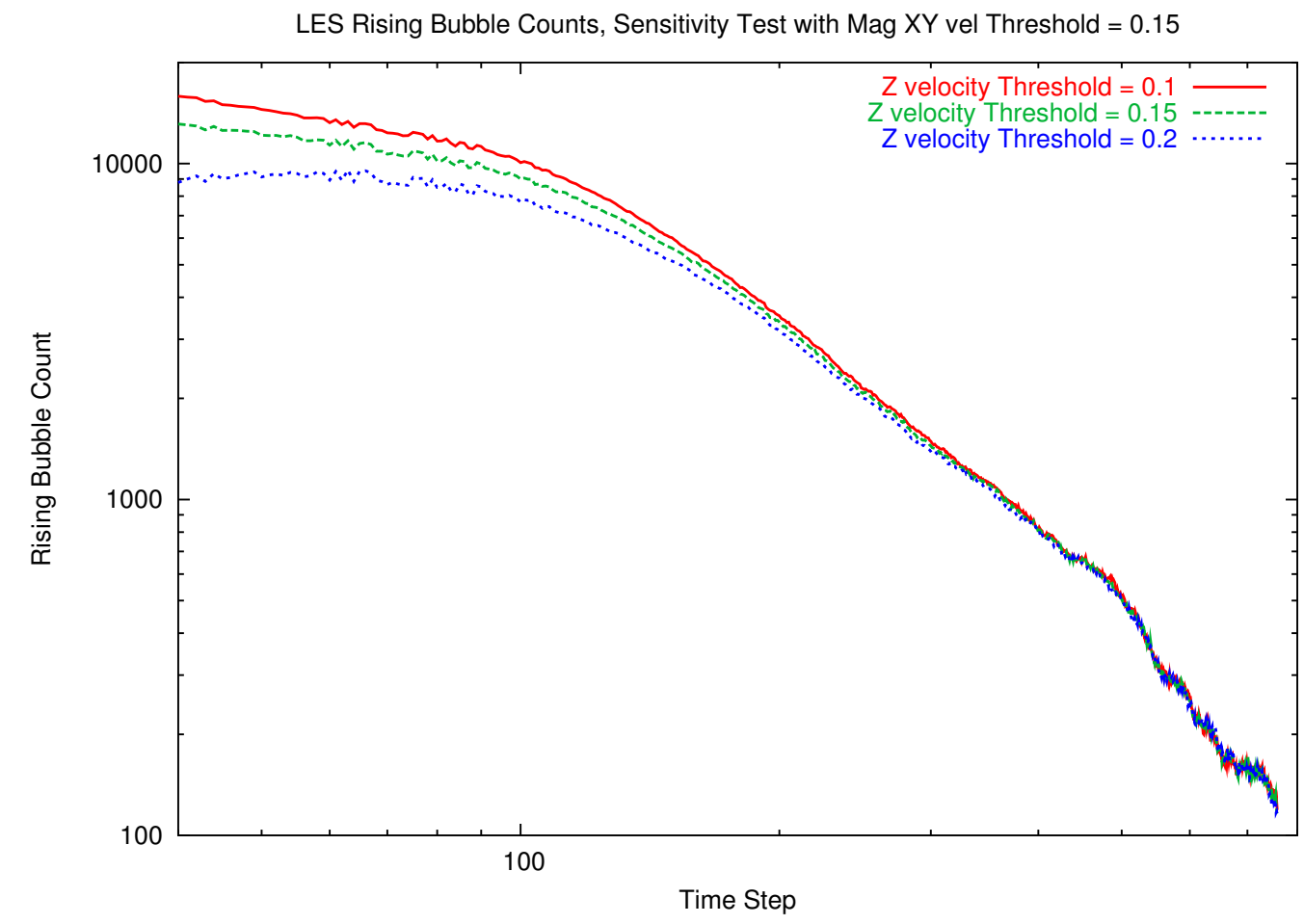

(a)

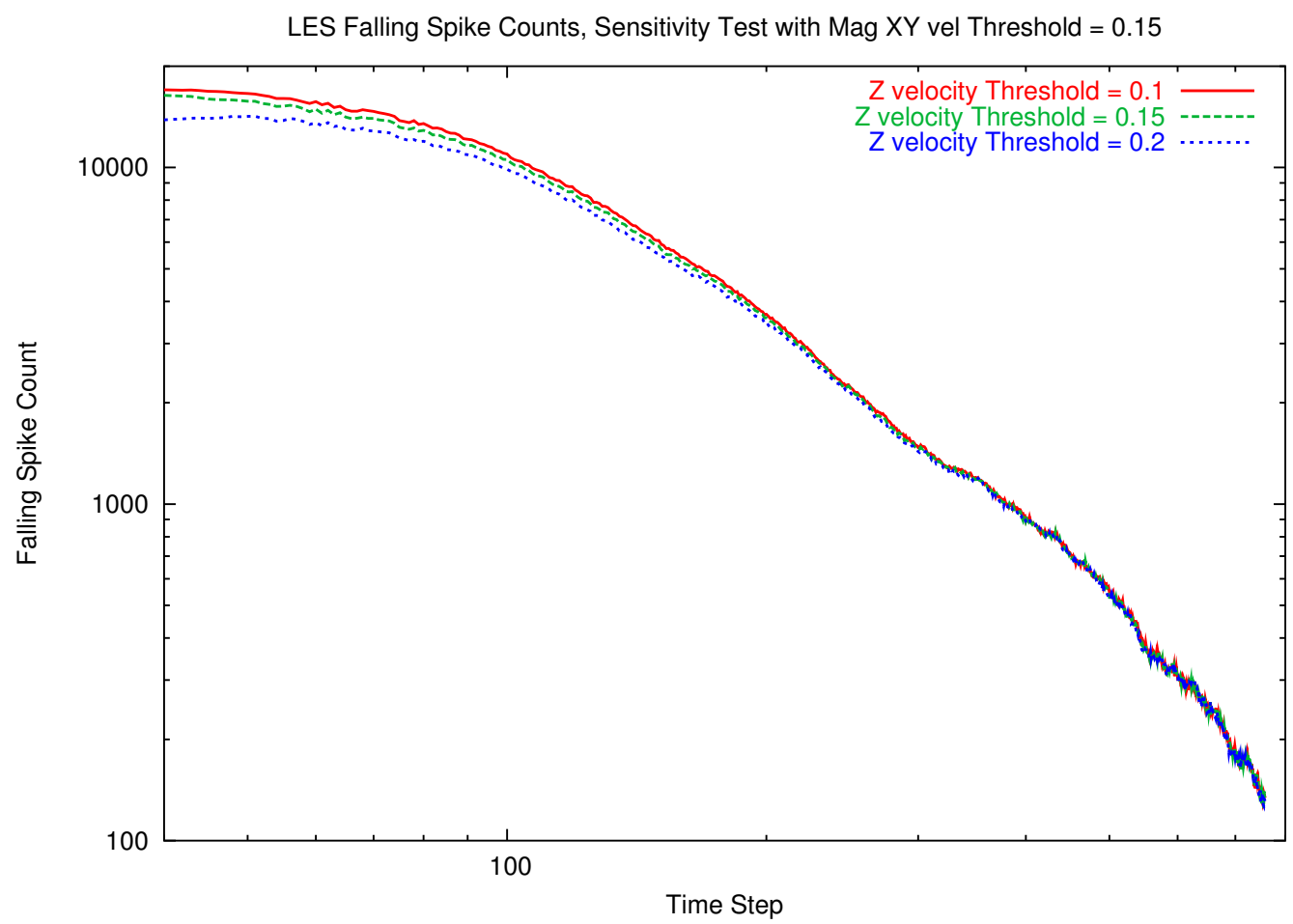

(b)

Figure 10: Effect of varying the $z$-velocity threshold on the rising bubble and falling spike counts for the LES data. The threshold on the magnitude of the $x-y$ velocity is set to 0.15. (a) Rising bubble count and (b) falling spike count. 
We observe that the rising bubbles and falling spike count curves also exhibit the four regimes we had seen earlier [4], though in some cases, such as the DNS and LES bubbles, the distinction between regimes 2 and 3 is not as clear as in the case of the spikes. We next analyze these curves further.

\subsection{Slopes for the bubble- and spike-count plots}

We next present the slopes of the rising bubble and falling spike counts for the four regimes for the LES and DNS data sets in Figures 13 and 14. The intent is to see if the counts exhibit a power-law behavior. In the figures, the end points of each regime are indicated by vertical lines and both the $\mathrm{x}$ - and the $\mathrm{y}$-axes are in log scale. The slopes were obtained after smoothing the curve and identifying regions where the curve was straight. This can be a challenge at later time steps, especially for the LES data, as the curves can be very noisy.

We observe from the figures that many of the slope values are close to integers. The exception is the slope of regime 4 for the LES bubbles, which may have been influenced by the fact that we did not include the tail end of the curve in the calculation of the slope as it is very noisy. Also, for the bubble curves for both DNS and LES, it is difficult to visually distinguish between regimes 2 and 3. Figure 15 shows the slopes for these curves when regimes 2 and 3 are merged into one. In contrast, for the spike curves for both the DNS and LES data, there is a clear difference between regimes 2 and 3, though for the LES spikes, the slopes in the two regimes are very similar.

\subsection{Average height of the rising bubbles and falling spikes}

We also calculate the height of the rising bubbles, and correspondingly, the depth of the falling spikes, using the definition proposed by Kartoon et al. in [5]. They consider the bubble front height to be the average over the tips of the rising bubbles.

In our case, for each bubble (spike), we define the tip to be the pixel with the highest height (depth) among those pixels which satisfy the requirements on the two thresholds for the bubbles (spikes). Figures 16 and 17 show the average bubble heights and spike depths for the DNS and LES data sets, respectively. We also include the heights (depths) for the bubbles (spikes) at 1\% and 5\% abundances obtained using a threshold definition [2]. The depth of a spike at $n \%$ is defined as the depth at which the average mole fraction of the heavy liquid is $n$. The corresponding bubble height at $\mathrm{n} \%$ is the height where the average mole fraction of the heavier fluid is $(1-n)$. [TODO - the previous description is likely incorrect.]

These plots indicate that the average bubble heights for the DNS and LES datasets match those obtained from the threshold definition at $5 \%$ abundance. For the spikes however, the curve obtained for the average spike depth lies almost midway in between the curves for the $1 \%$ and $5 \%$ abundances.

\subsection{Comparison with counts for all bubbles and spikes}

Finally, we compare the counts of the rising bubbles and falling spikes with the counts of all bubbles and all spikes from our earlier work [4]. For the latter, we consider the counts obtained using the magnitude of the $x-y$ velocity with the variance criterion. Figures 18 and 19 show how the counts for all bubbles (spikes) compare with the counts for the rising bubbles (falling spikes). As in the paper by Kartoon at al. [5], we also include the ratio of the rising bubbles (falling spikes) to all bubbles (spikes). 


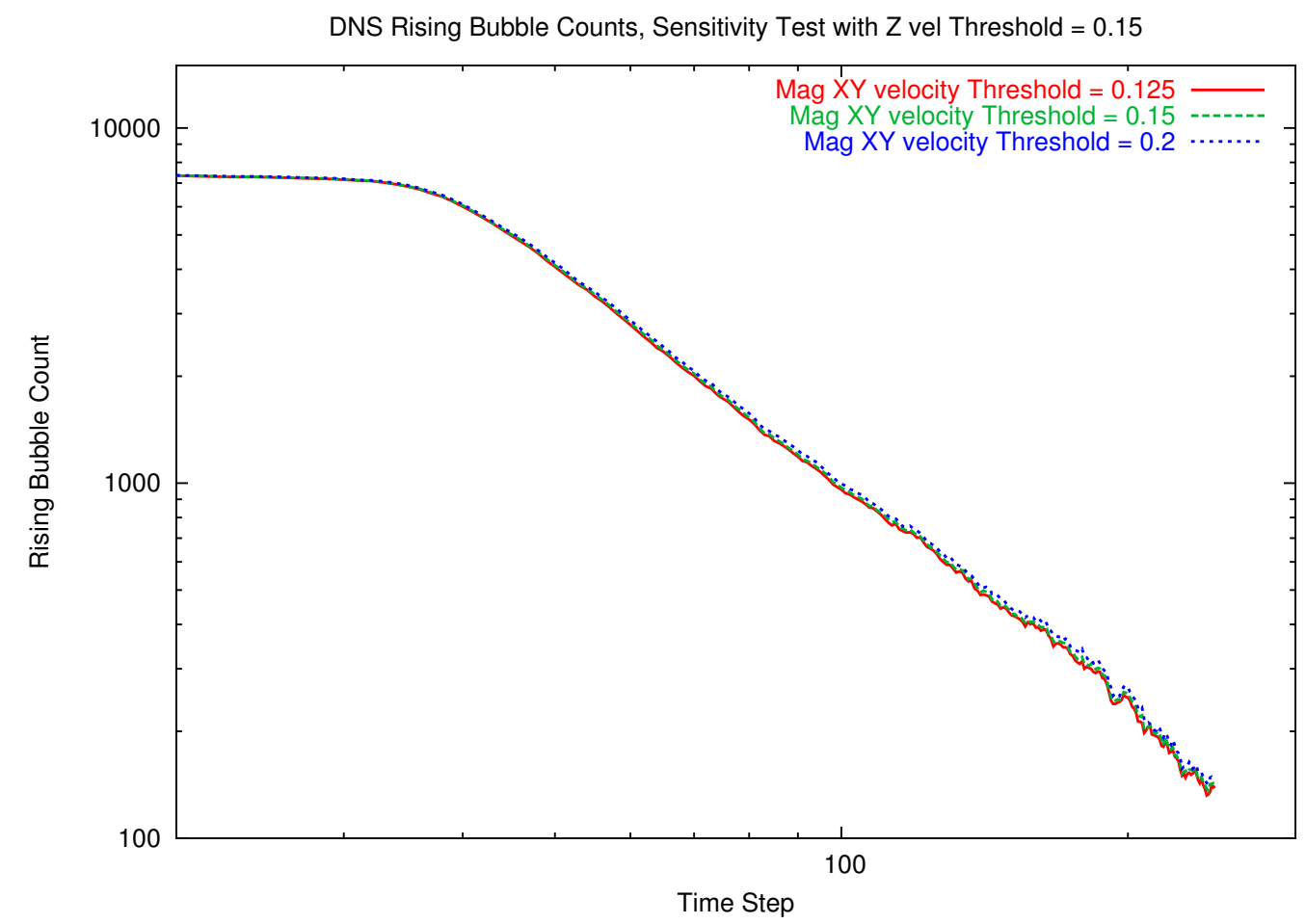

(a)

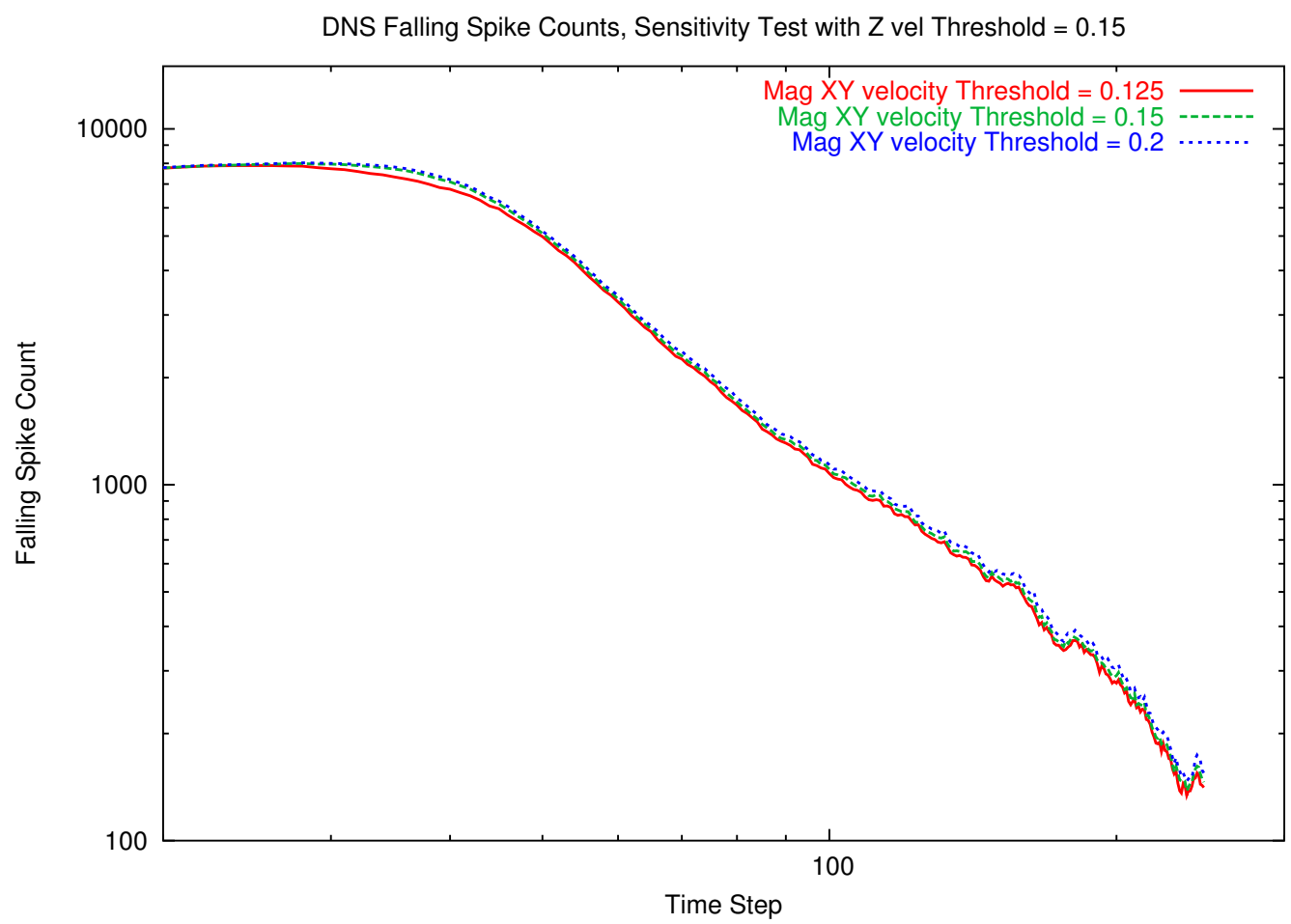

(b)

Figure 11: Effect of varying the threshold on the magnitude of the $x$ - $y$ velocity on the rising bubble and falling spike counts for the DNS data. The threshold on the $z$-velocity is set to 0.15. (a) Rising bubble count and (b) falling spike count. 


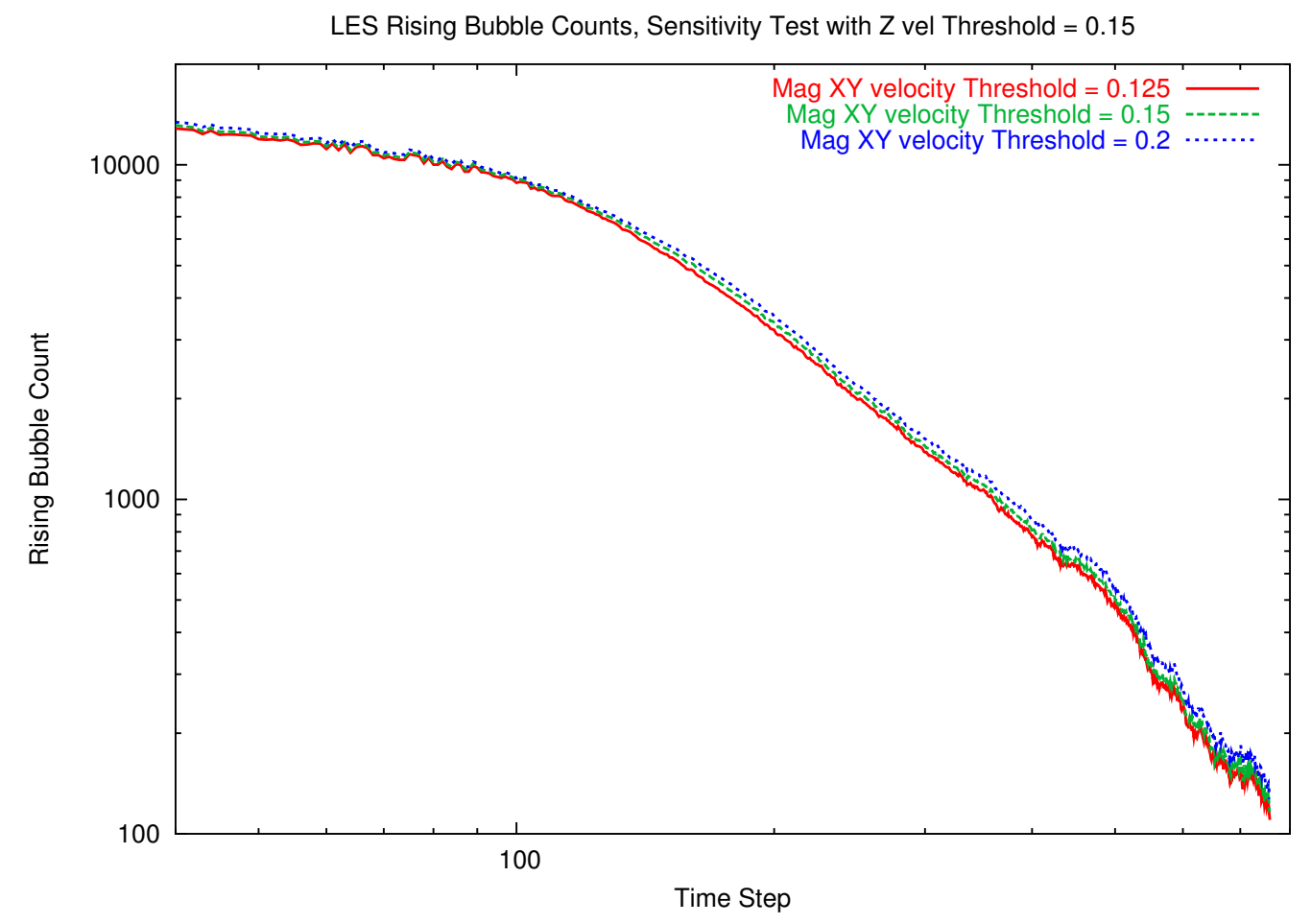

(a)

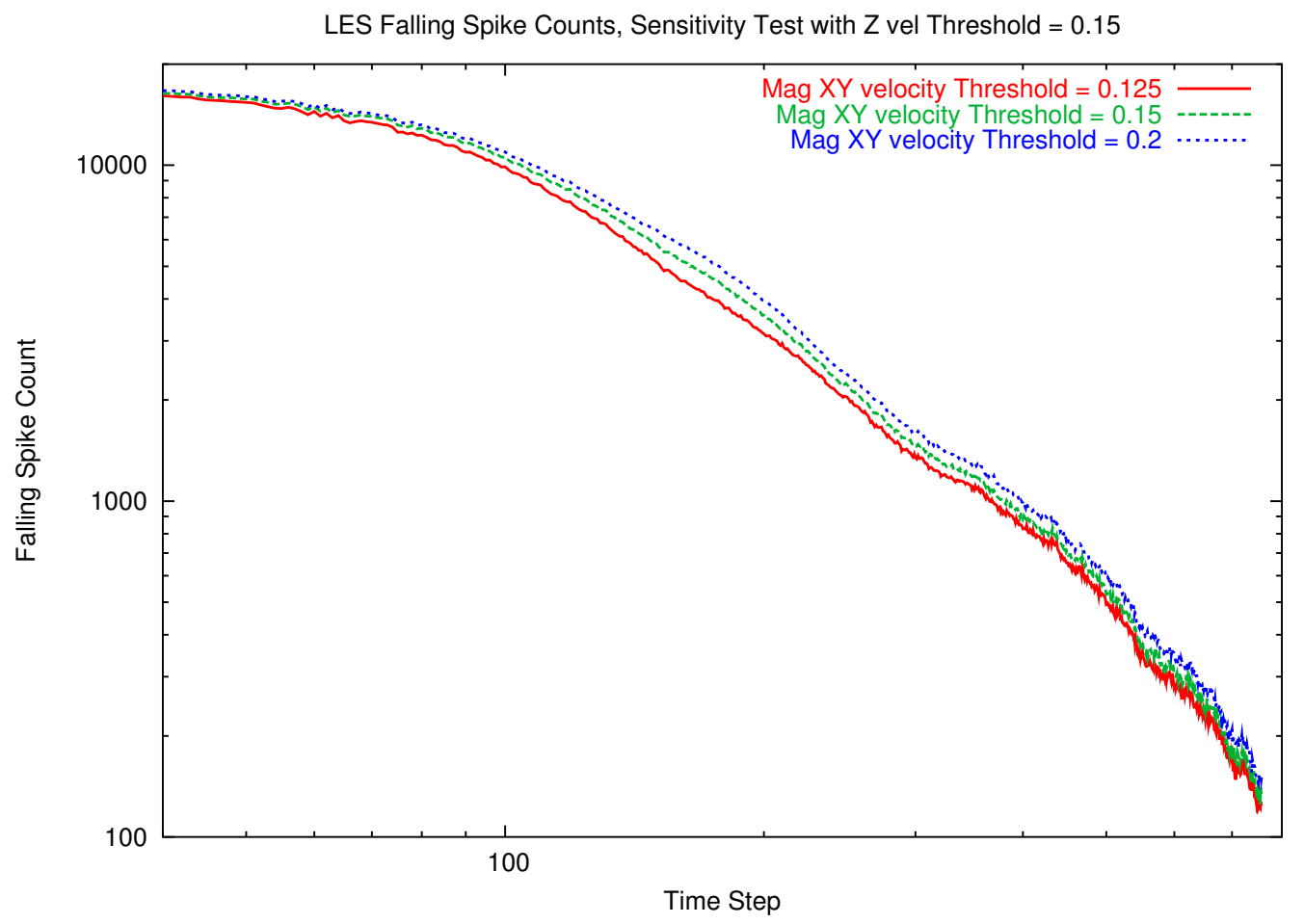

(b)

Figure 12: Effect of varying the threshold on the magnitude of the $x-y$ velocity on the rising bubble and falling spike counts for the LES data. The threshold on the $z$-velocity is set to 0.15. (a) Rising bubble count and (b) falling spike count. 


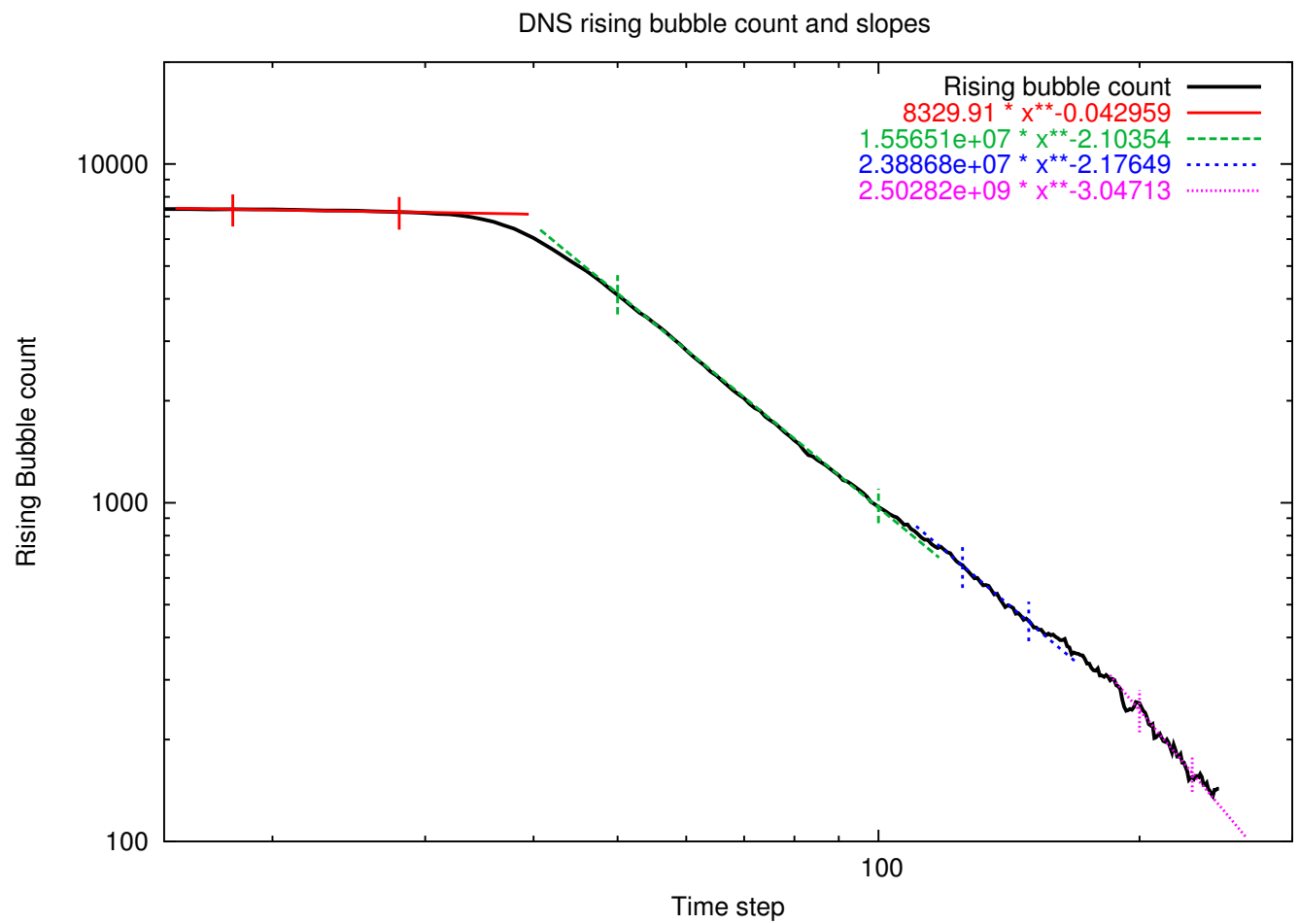

(a)

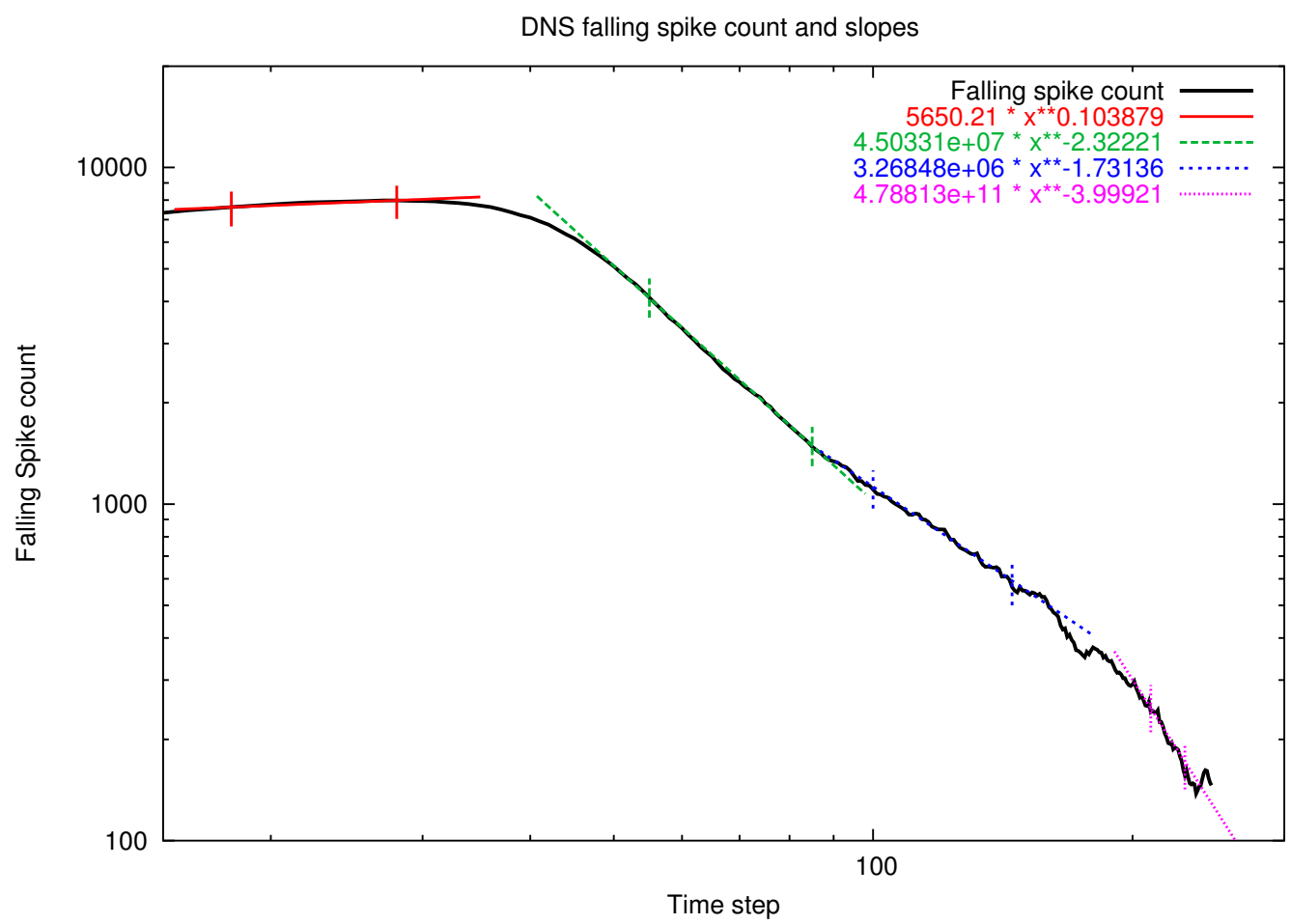

(b)

Figure 13: The slopes for the four regimes in the rising bubble and falling spike counts for the DNS data. The counts are obtained using $T_{z}=0.15$ and $T_{x-y}=0.15$. (a) Rising bubble count and (b) falling spike count. 


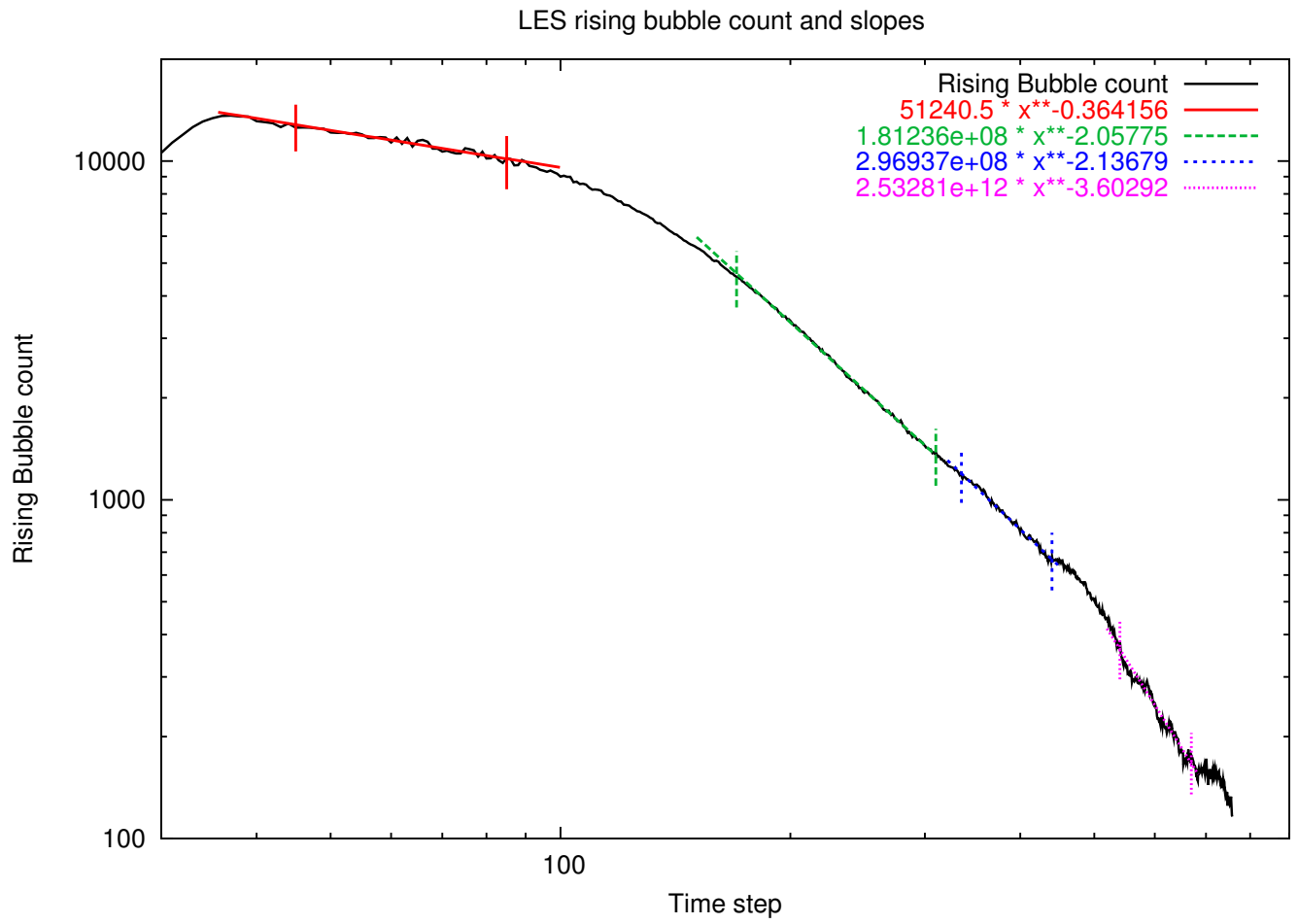

(a)

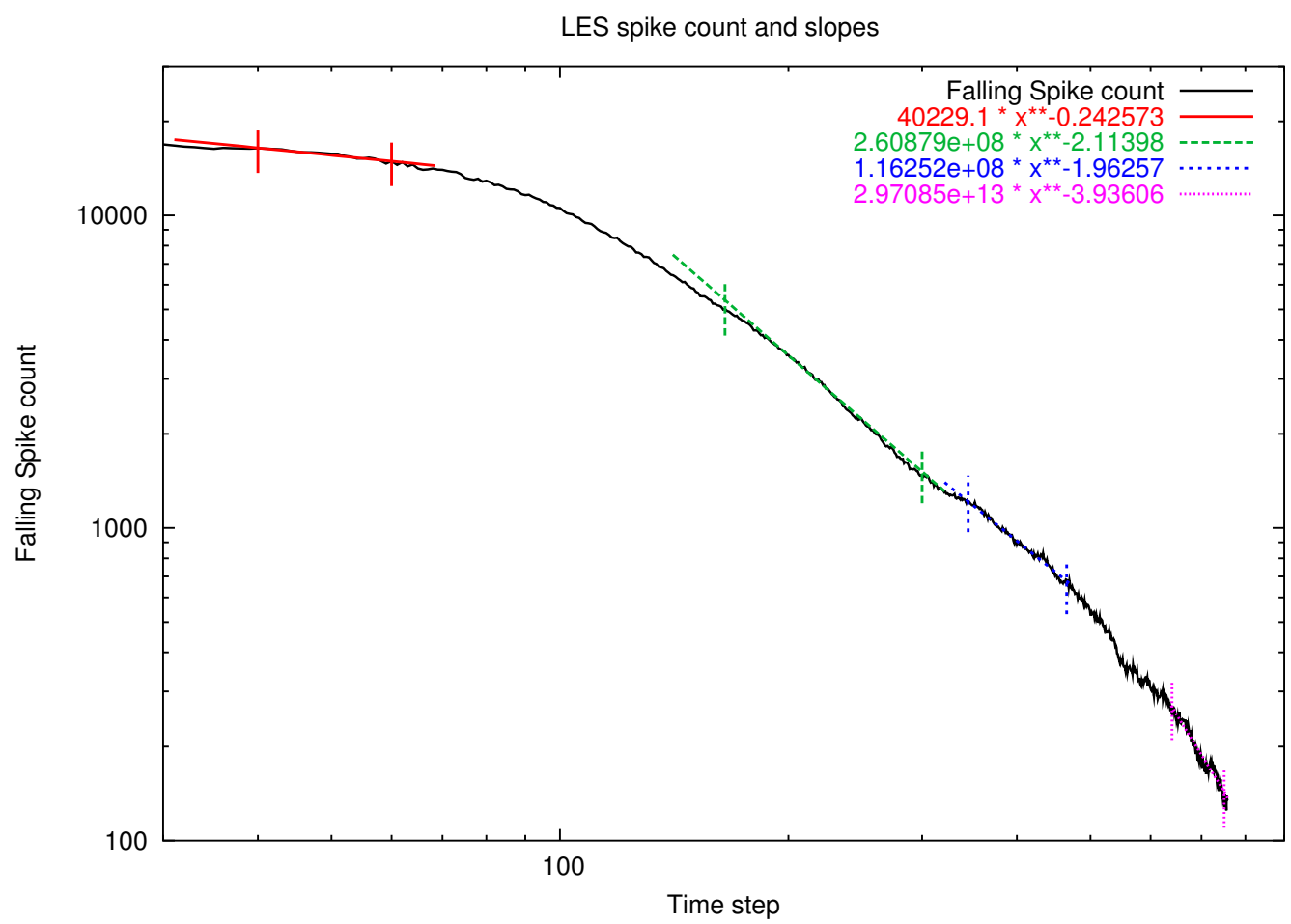

(b)

Figure 14: The slopes for the four regimes in the rising bubble and falling spike counts for the LES data. The counts are obtained using $T_{z}=0.15$ and $T_{x-y}=0.15$. (a) Rising bubble count and (b) falling spike count. 


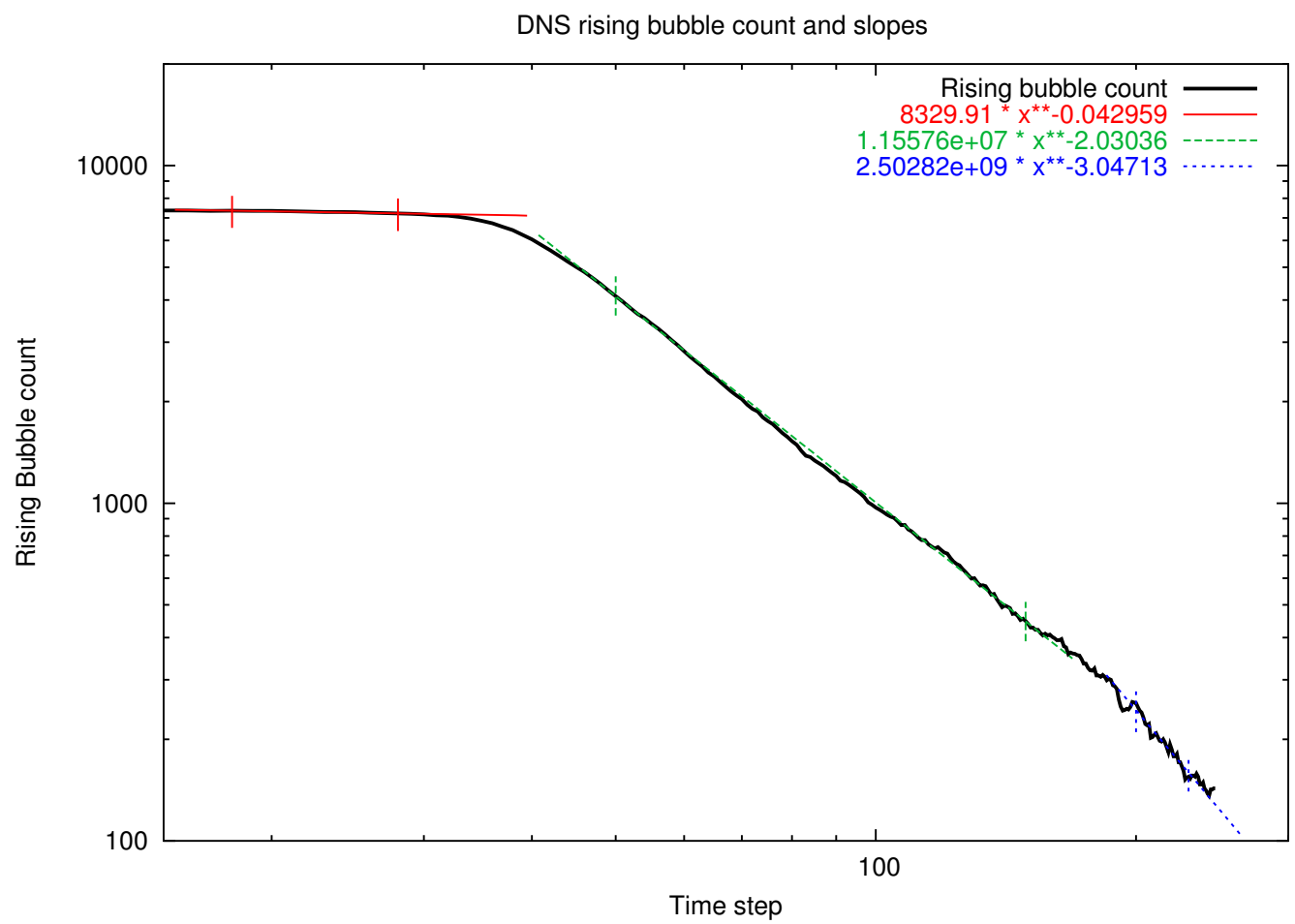

(a)

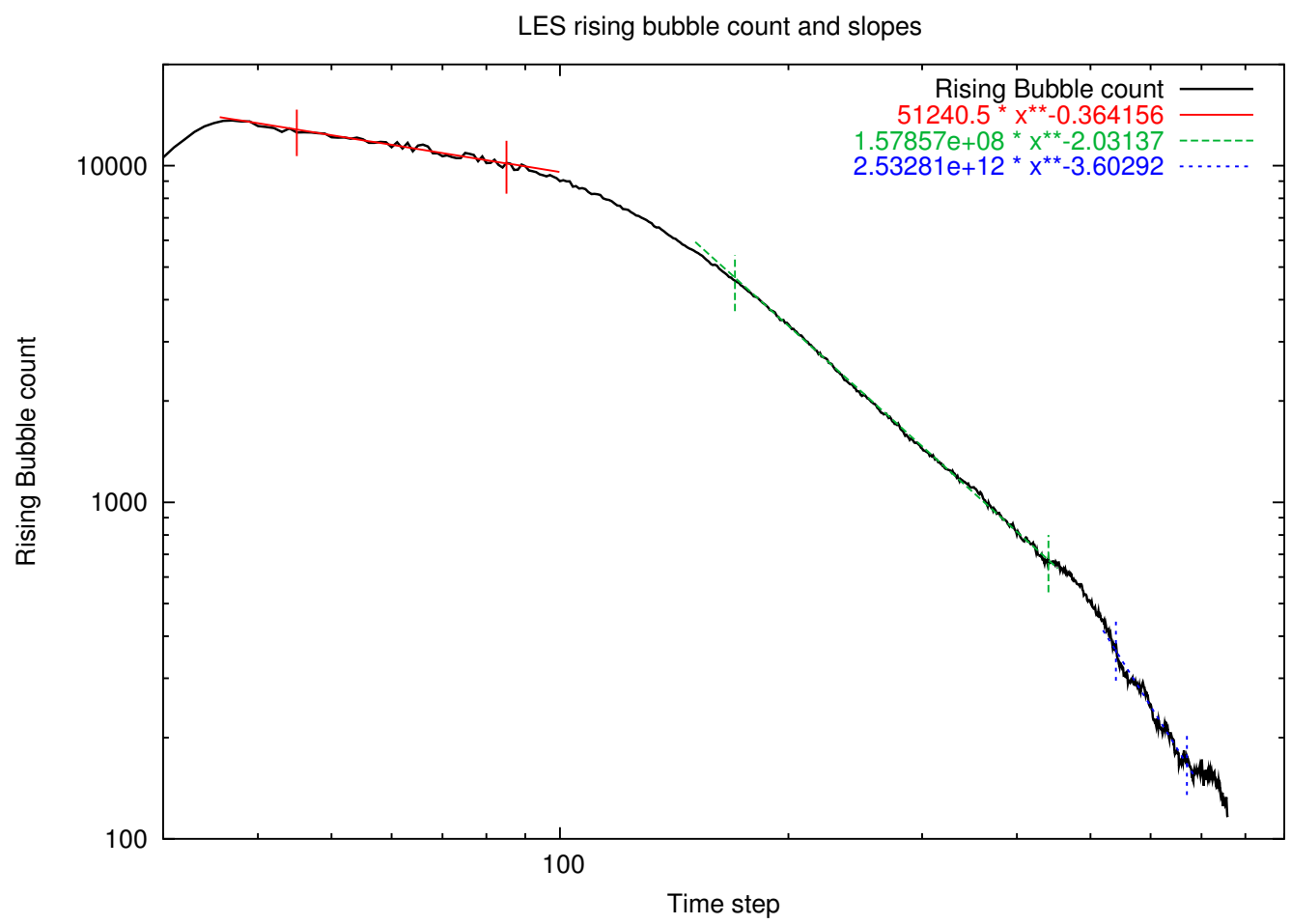

(b)

Figure 15: The slopes for rising bubble counts for (a) the DNS data and (b) the LES data. These plots merge regimes 2 and 3 into one. The counts are obtained using $T_{z}=0.15$ and $T_{x-y}=0.15$. 


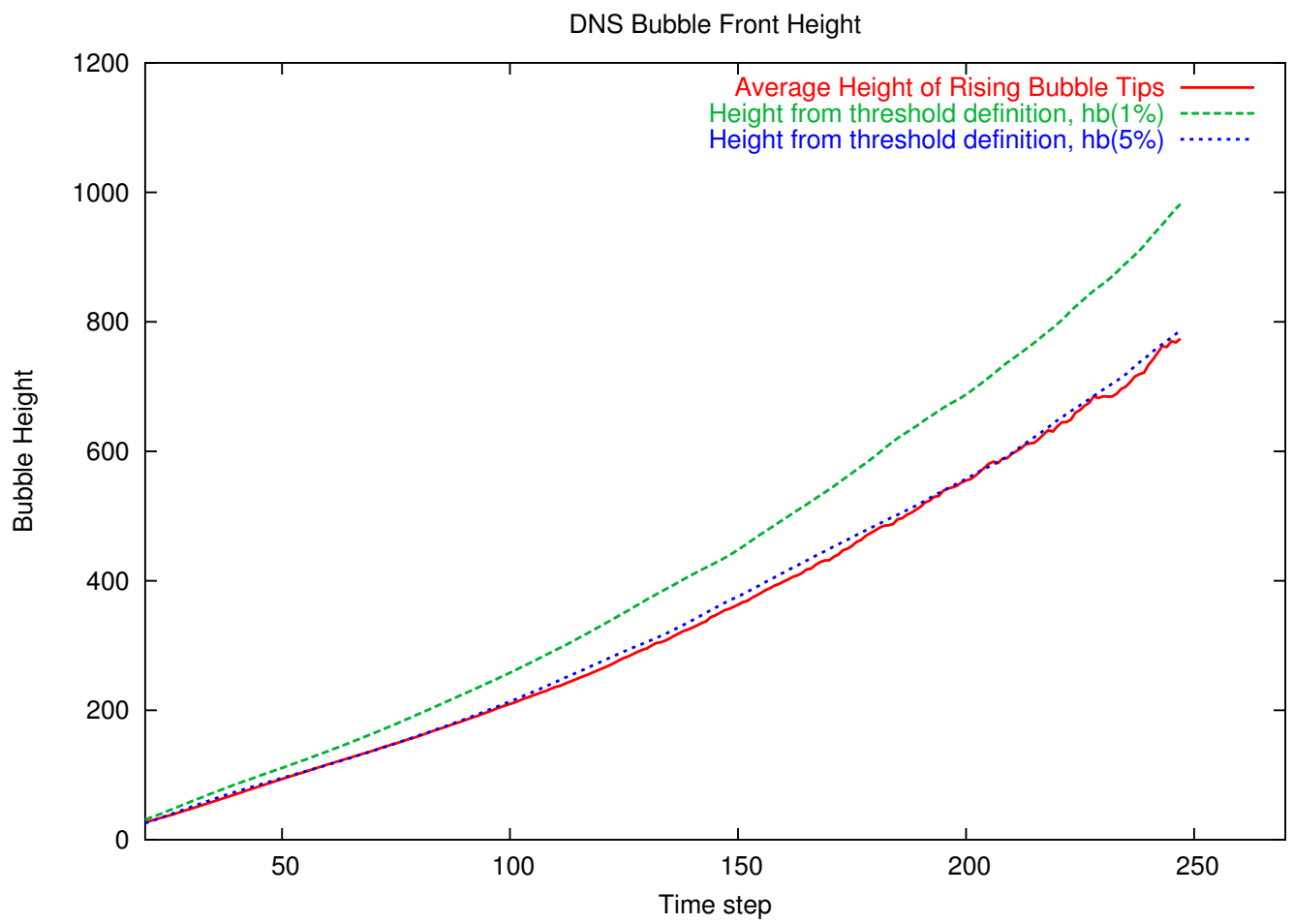

(a)

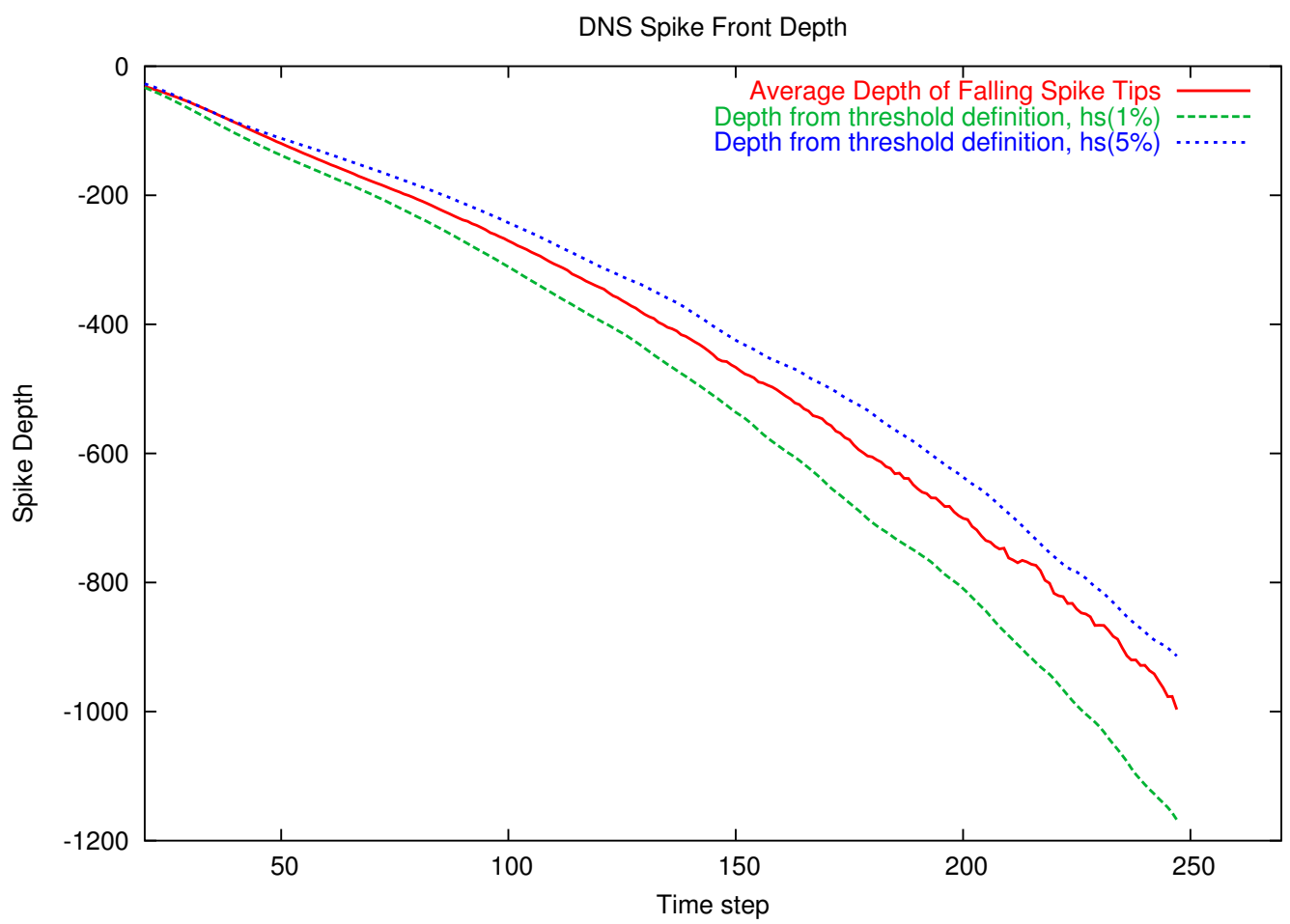

(b)

Figure 16: (a) The average rising bubble height and (b) the falling spike depth for the DNS data. The results are obtained using $T_{z}=0.15$ and $T_{x-y}=0.15$. Also included are the curves obtained using the threshold definition at $1 \%$ and $5 \%$ abundances. 


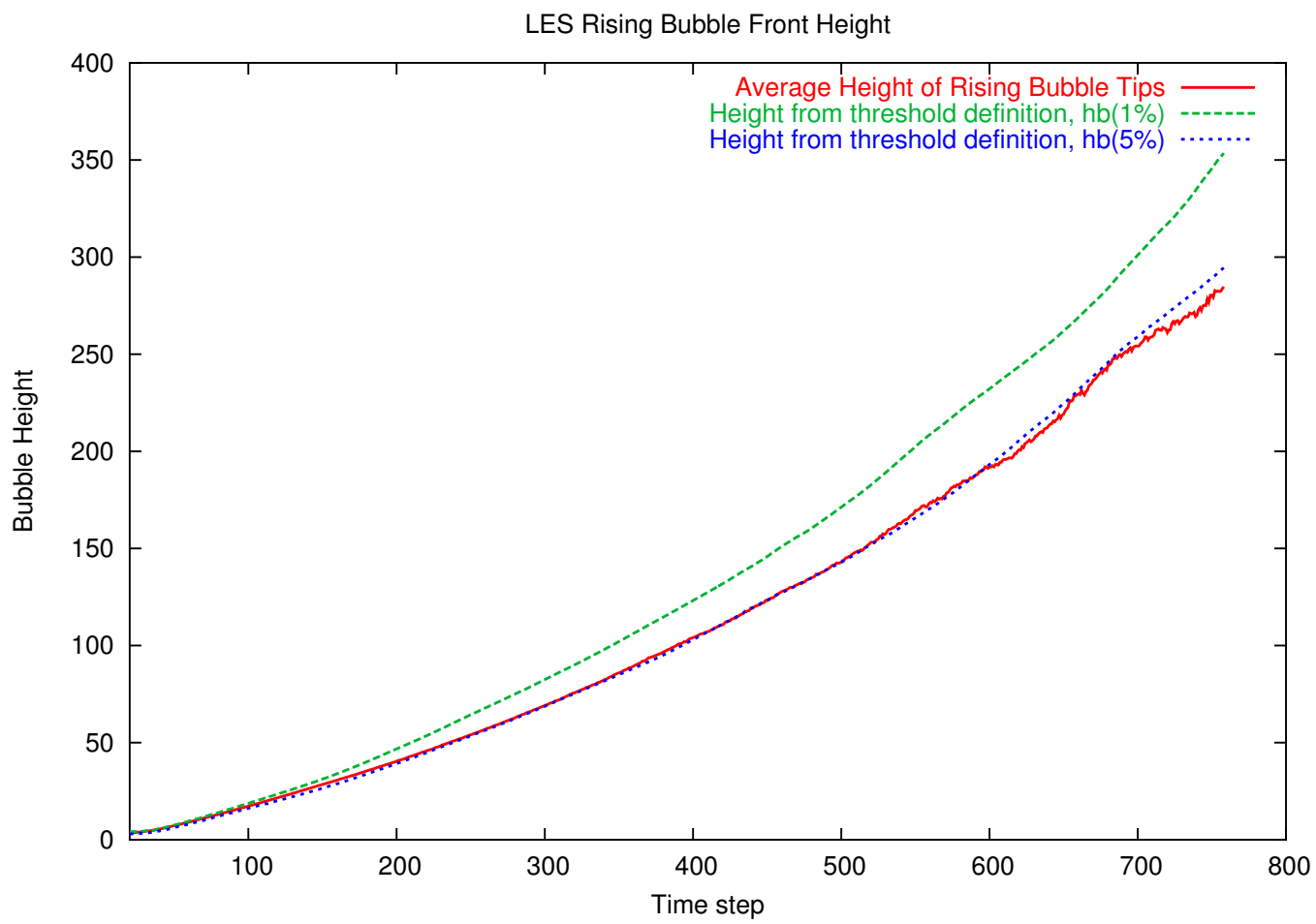

(a)

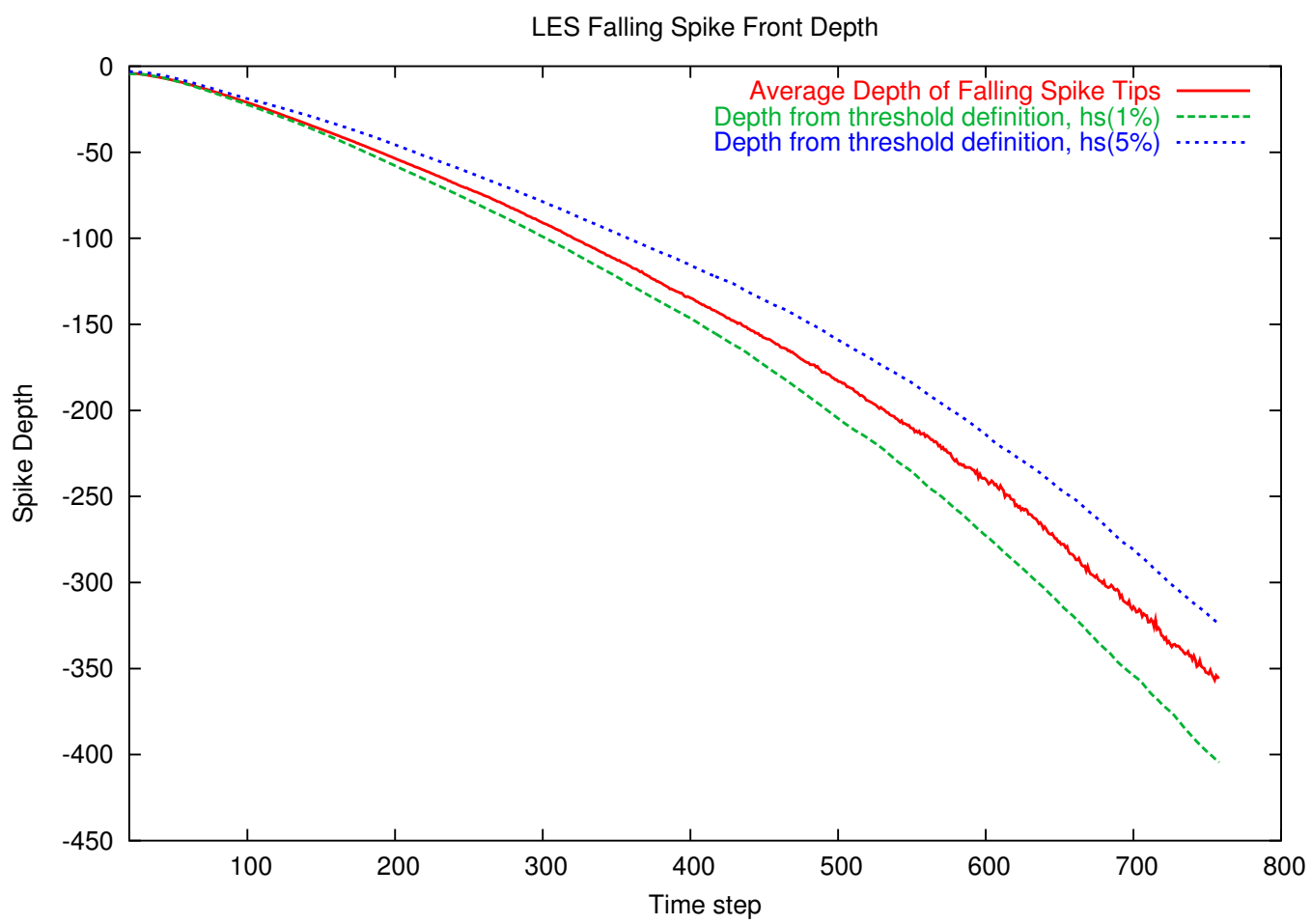

(b)

Figure 17: (a) The average rising bubble height and (b) the falling spike depth for the LES data. The results are obtained using $T_{z}=0.15$ and $T_{x-y}=0.15$. Also included are the curves obtained using the threshold definition at $1 \%$ and $5 \%$ abundances. 


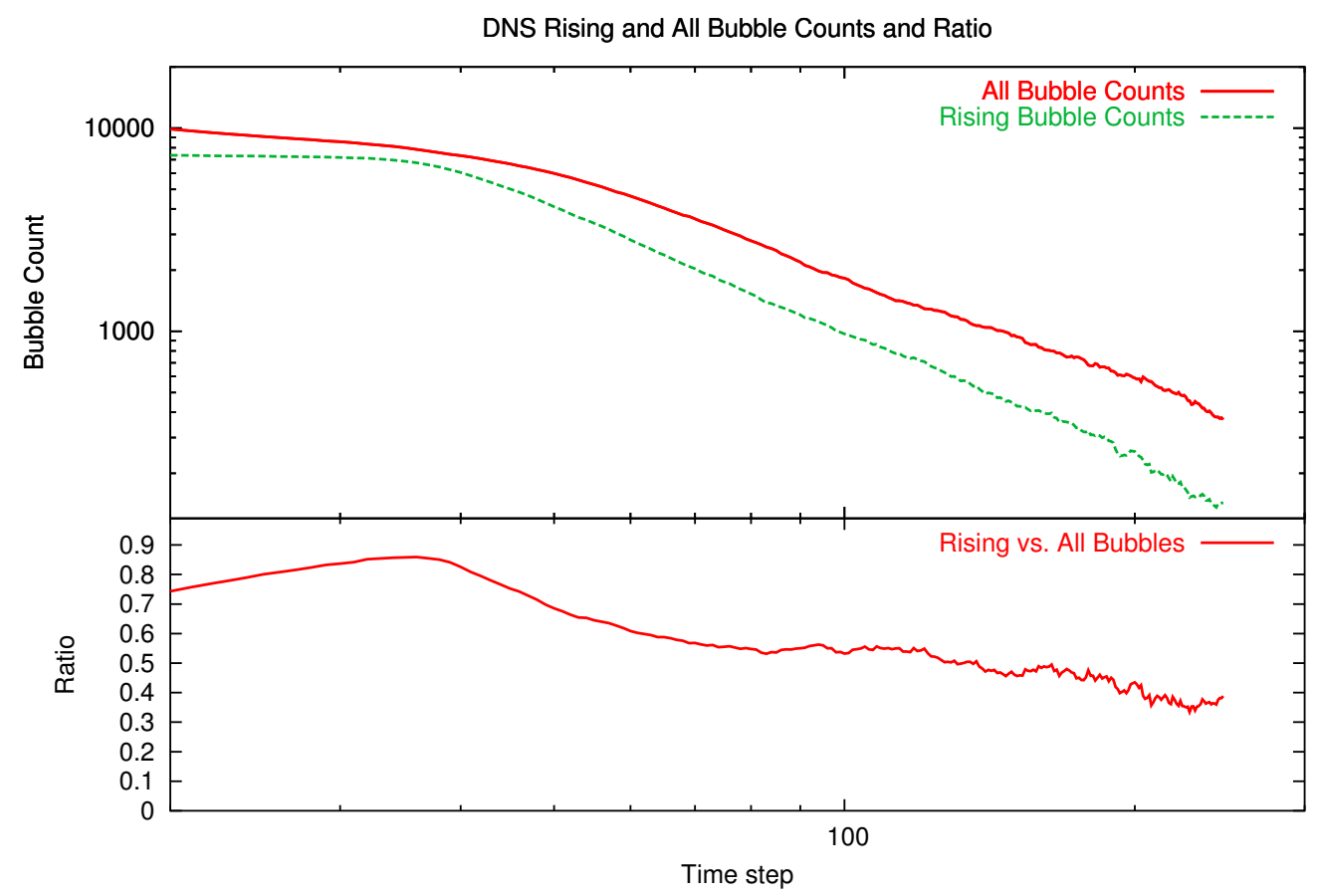

(a)

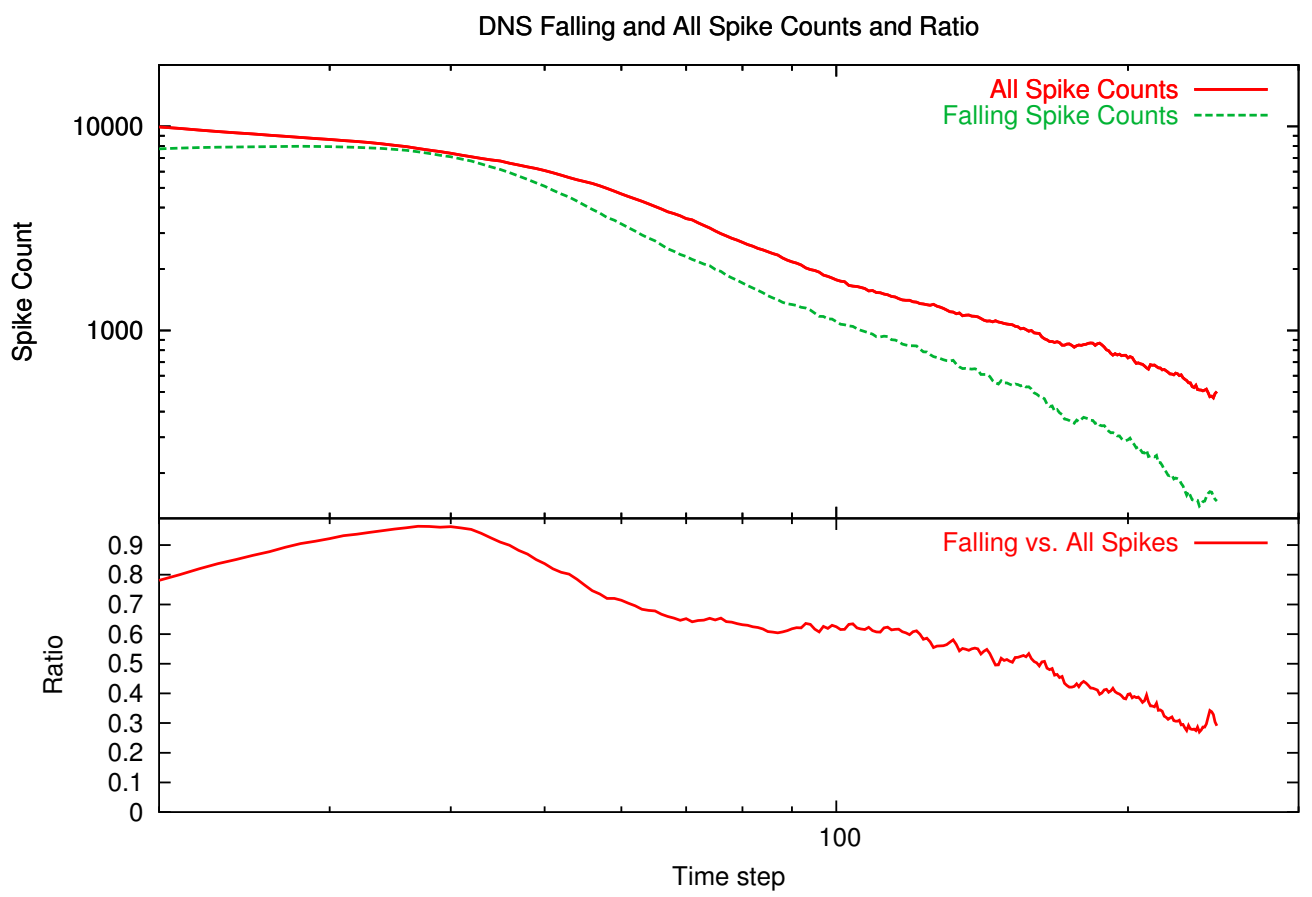

(b)

Figure 18: (a) The ratios for rising bubble count to all bubble count and (b) falling spike count to all spike count for the DNS data. The rising bubble and falling spike results are obtained using $T_{z}=0.15$ and $T_{x-y}=0.15$. The all bubble and spike counts are obtained using the magnitude of the $x-y$ velocity with the variance criterion. 
LES Rising and All Bubble Counts and Ratio

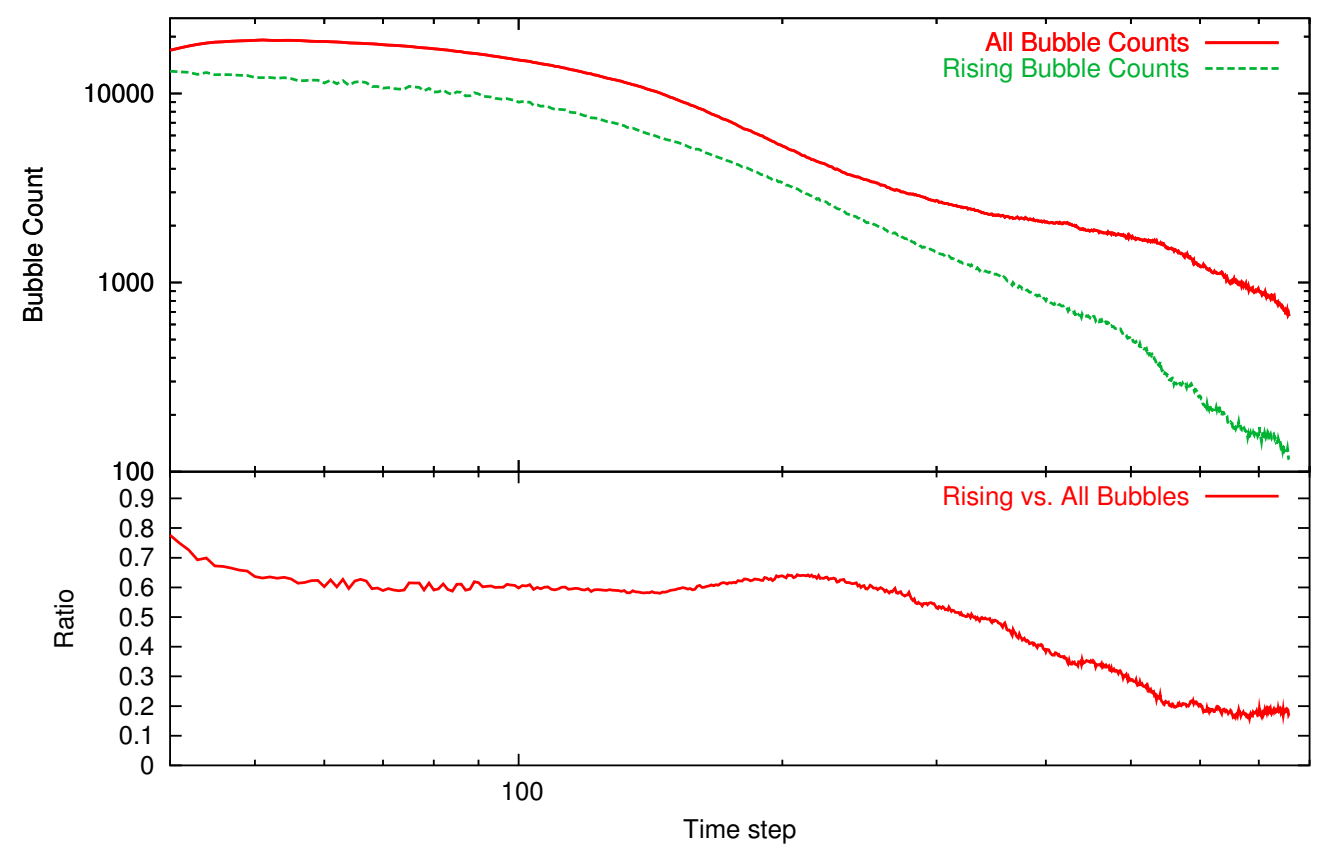

(a)

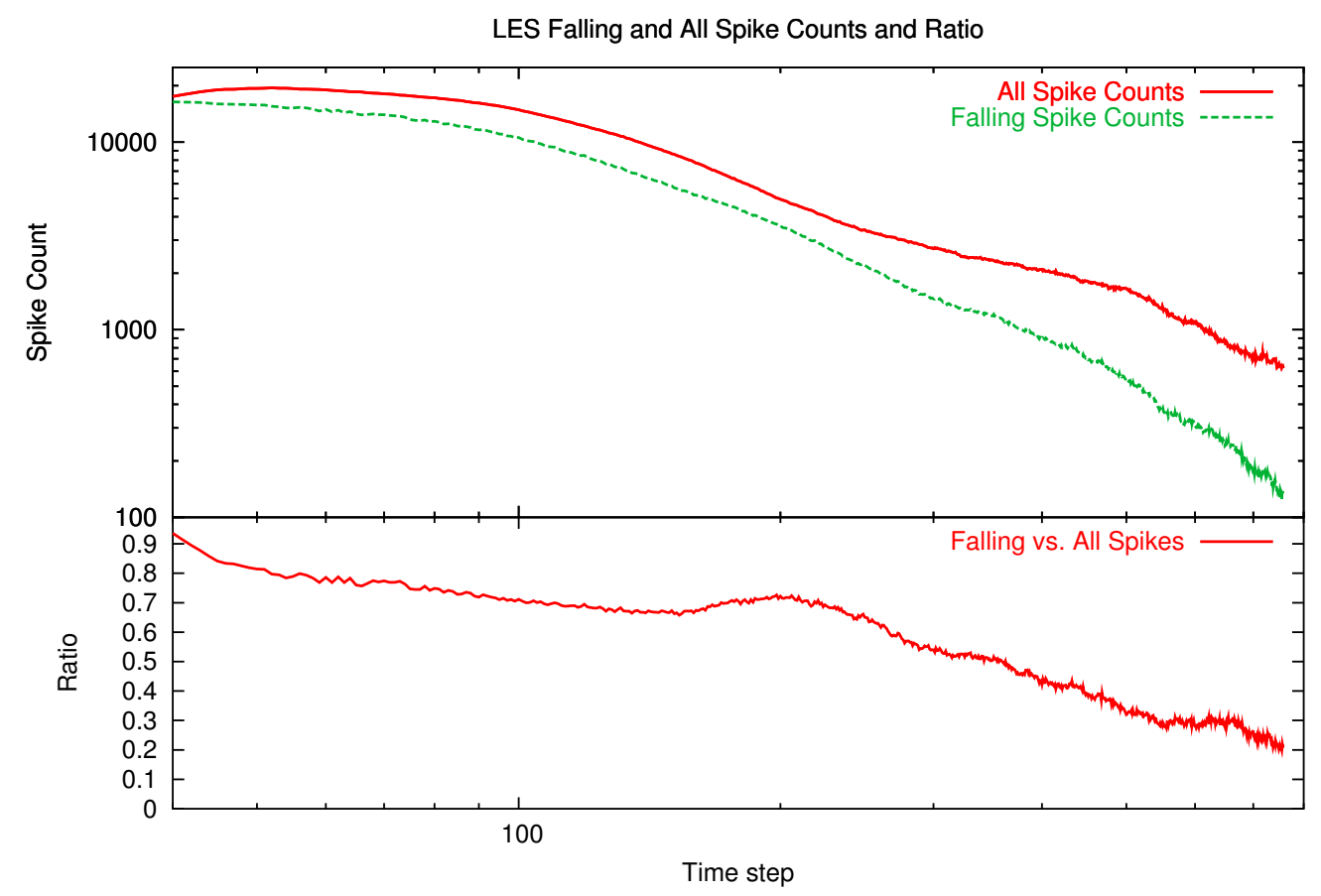

(b)

Figure 19: (a) The ratios for rising bubble count to all bubble count and (b) falling spike count to all spike count for the LES data. The rising bubble and falling spike results are obtained using $T_{z}=0.15$ and $T_{x-y}=0.15$. The all bubble and spike counts are obtained using the magnitude of the $x-y$ velocity with the variance criterion. 
We observe the following regarding these ratios. For the DNS bubbles, the percentage of rising bubbles increases initially as expected in the first regime where the bubbles grow independently. After reaching a peak of ratio of 0.85 at around time step 35, the percentage of rising bubbles starts to reduce when the bubbles start competing with each other. The ratio remains around 0.5 for most of regimes 2 and 3 as indicated by ratios in the range [0.45, 0.55] between time steps 80 to 190 , after which the ratio reduces to 0.35 . The DNS spikes show similar behavior, though the peak ratio is 0.96 at early time, with the falling spikes accounting for most of the spikes. Between time steps 70 through 140, the ratio is in the range [0.55, 0.65], and reduces to 0.3 at late time.

The ratios for the LES data have a different behavior from that of the DNS data. There is no inital phase of increasing ratio as in the case of the DNS data; instead, there is a long, almost flat, region in the first half, followed by a small bump, and then declining ratios in the second half of the simulation. For the LES bubbles, in the first half, the ratio is around 0.6 till nearly time step 300 after which it reduces gradually to nearly 0.2 at late time. The spikes ratios are similar, except they reach a value of 0.7 in the first half and reduce to 0.25 at late time.

For both the LES and DNS data, the ratios for the spikes are higher than for the bubbles, though the overall structure of the bubble and spike ratio curves are very similar. We note that in the paper by Kartoon et al. [5], they observe a ratio of 0.5 for rising bubbles vs. all bubbles using their definition of a rising bubble.

\section{Conclusions}

In this paper, we extended our earlier work on the analysis of bubbles and spikes in two simulations of the Rayleigh-Taylor instability to focus on the rising bubbles and falling spikes. We considered several ways of constraining the count to a subset of all bubbles and spikes. We found that a technique which first identified rising (falling) regions based on the $z$-velocity, and then considered the regions which also satisfied the bubble (spike) definition based on the magnitude of the $x-y$ velocity, was robust and insensitive to the choice of threshold parameters.

We extracted several statistics on the rising bubbles and falling spikes. As in our earlier work, we found that the counts indicated four regimes in the process of the mixing of the two fluids. However, in this case, we found that the slopes of the regimes were close to integer values. Further, the average height (depth) of the rising bubbles (spikes) was related to the corresponding values obtained using a more common threshold-based definition. A comparison of these new results with the earlier counts indicated that for the DNS (LES) bubble data, the ratio of rising bubbles to all bubbles was around 0.5 (0.6) for a large part of the mixing process. The corresponding ratios for the DNS (LES) spikes are 0.6 (0.7). We also observed that for both the DNS and the LES data, the curves for the bubble and spike ratios are very similar, with the spike curves having higher ratios than the bubble curves.

Our definition of the rising bubbles and falling spikes also allows us to track these structures over time to understand the dynamics of the mixing of the fluids. In addition, it allows us to approximate the sizes of the bubbles, at least at early time. We shall explore these ideas in future work. 


\section{Acknowledgment}

The data used in this work was generated by Andrew Cook and William Cabot, who graciously made it available for our analysis. William Cabot also provided the bubble heights and spike depths for the threshold-based definitions which were used in Section 4.4. The analysis was done using the Sapphire scientific data mining software developed at LLNL.

This work performed under the auspices of the U.S. Department of Energy by Lawrence Livermore National Laboratory under Contract DE-AC52-07NA27344.

\section{References}

[1] Cabot, W. H., And Cook, A. W. Reynolds number effects on Rayleigh-Taylor instability with possible implications for type Ia supernovae. Nature Physics 2 (2006), 562-568.

[2] Cook, A. W., Cabot, W. H., And Miller, P. L. The mixing transition in RayleighTaylor instability. Journal of Fluid Mechanics 511 (2004), 333-362.

[3] Gardner, L., Glimm, J., McBryan, O., Menikoff, R., Sharp, D. H., and Zhang, Q. The dynamics of bubble growth for Rayleigh-Taylor unstable interfaces. Physics of Fluids 31, 3 (1988), 447-465.

[4] Kamath, C., Gezahegne, A., And Miller, P. L. Analysis of Rayleigh-Taylor instability, Part I: Bubble and spike count. Tech. Rep. UCRL-TR-223676, Lawrence Livermore National Laboratory, 2006. http://www.1lnl.gov/casc/sapphire/pubs/TR-223676.pdf.

[5] Kartoon, D., Oron, D., Arazi, L., and Shvarts, D. Three-dimensional multimode Rayleigh-Taylor and Richtmyer-Meshkov instabilities at all density ratios. laser and Particle Beams 21 (2003), 327-334.

[6] Sharp, D. H. An overview of Rayleigh-Taylor instability. Physica 12D (1984), 3-18.

[7] Sonka, M., Hlavac, V., And Boyle, R. Image Processing, Analysis, and Machine Vision. PWS Publishing, 1999. 